\title{
Groupoids associated to inverse semigroups
}

\author{
by \\ Malcolm Jones
}

A thesis

submitted to the Victoria University of Wellington

in fulfilment of the

requirements for the degree of

Master of Science

in Mathematics.

Victoria University of Wellington

2020 



\begin{abstract}
Starting with an arbitrary inverse semigroup with zero, we study two wellknown groupoid constructions, yielding groupoids of filters and groupoids of germs. The groupoids are endowed with topologies making them étale. We use the bisections of the étale groupoids to show there is a topological isomorphism between the groupoids. This demonstrates a widely useful equivalence between filters and germs. We use the isomorphism to characterise Exel's tight groupoid of germs as a groupoid of filters, to find a nice basis for the topology on the groupoid of ultrafilters and to describe the ultrafilters in the inverse semigroup of an arbitrary self-similar group.
\end{abstract}




\section{Acknowledgments}

The results of this project were not achieved in isolation.

My supervisors, Lisa Orloff Clark and Astrid an Huef, and collaborators, Becky Armstrong and Ying-Fen Lin, have contributed enormously to my learning in a way that has enabled me to grow both as a student and as an individual.

Behind the scenes there has been a supportive community of staff and students in the School of Mathematics and Statistics, Te Kura Mātai Tatauranga. Their efforts to maintain our academic home are too often thankless. Thank you.

Laslty, I acknowledge friends and whānau for all the times they indulged me while I spouted jargon and the times they inspired me to think differently and creatively about mathematics. 


\section{Contents}

$\begin{array}{lll}1 & \text { Introduction } & 1\end{array}$

$\begin{array}{|ll|}2 & \text { Inverse semigroups }\end{array}$

$2.1 \quad$ Inverse semigroups . . . . . . . . . . . . . . . 5

2.1 .1 Preliminaries . . . . . . . . . . . . . . . 5

$2.1 .2 \quad$ Assuming zero exists $\ldots \ldots \ldots \ldots$

$2.1 .3 \quad$ Natural partial order . . . . . . . . . . . . . . 10

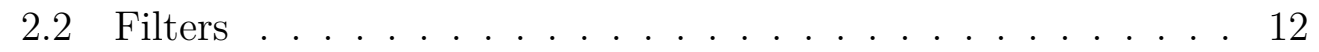

2.2 .1 Trivial and proper filters . . . . . . . . . . . 13

2.2 .2 Ultrafilters . . . . . . . . . . . . . . . . . 14

$2.2 .3 \quad$ Idempotent filters and filters of idempotents . . . . . . 15

$2.2 .4 \quad$ Inverse semigroup of filters . . . . . . . . . . 16

\begin{tabular}{lll}
\hline 3 & Groupoids & 21
\end{tabular}

$3.1 \quad$ Groupoids of filters and germs . . . . . . . . . . . . . . . . . 21

$3.1 .1 \quad$ Groupoids associated to inverse semigroups. . . . . . . 21

3.1 .2 Groupoids of filters . . . . . . . . . . . . . . . 23

3.1 .3 Groupoids of germs . . . . . . . . . . . . . . . 27

3.2 Étale groupoids of filters and germs . . . . . . . . . . . . 30

3.2 .1 Topologies on filters of idempotents . . . . . . . . . . 30

3.2 .2 Patch topological groupoid of filters . . . . . . . . . . . 34

$3.2 .3 \quad$ Étale groupoids . . . . . . . . . . . . . . . . . 37

3.3 Hausdorff unit spaces . . . . . . . . . . . . . . . . . . . . . . . 42 
3.4 Filters isomorphic to germs . . . . . . . . . . . . . . . . . . 44 $3.4 .1 \quad$ Ultrafilters . . . . . . . . . . . . . . . . . . . . . . . . . 48

3.4 .2 Exel's tight groupoid . . . . . . . . . . . . . . . . . . . 49

3.5 Application to self-similar groups . . . . . . . . . . . . 50

$3.5 .1 \quad$ Filters in terms of filters of idempotents . . . . . . . 50

$3.5 .2 \quad$ Filters associated to self-similar groups . . . . . . . . . 51 


\section{Chapter 1}

\section{Introduction}

In this thesis we investigate various groupoids associated to inverse semigroups. Groupoids (introduced by Brandt [Bra27]) and inverse semigroups (introduced independently by Wagner Vag52 and Preston Pre54a; Pre54b Pre54c]) are generalisations of groups. The importance of groupoids in the study of operator algebra, in particular, is demonstrated in Renault's monograph [Ren80], where he describes how to associate a $\mathrm{C}^{*}$-algebra to a groupoid. Renault also poses the question of finding nice groupoids associated to inverse semigroups in [Ren80, Remark III.2.4]. Starting with an arbitrary inverse semigroup, we consider two well-known groupoid constructions and show that there is an isomorphism between the groupoids.

The first construction uses a 'filter approach'. A filter is a special subset of the inverse semigroup depending on the natural partial order. The filter approach was pioneered by Lenz in [Len08] and has seen refinement over time Law12, LMS13; Bic19; Cas20].

Another well-known groupoid construction is the 'germ approach', which depends on an action of the inverse semigroup on the space of representations of its idempotents. Paterson founded the germ approach [Pat99, pp. 177], which was streamlined by Exel in Exe08.

According to the literature, algebraists tend to use the filter approach while analysts use the germ approach. Thus, our work is partly motivated 
by an interest in unifying these two constructions to enable diverse collaborations.

It is mentioned in the literature that the two approaches yield the same groupoids up to isomorphism (for example, see [Res10, Exercise III.3.16(2)], [LMS13, §3.3], [LL13, §5.1], Bic19, Remark 2.41] and [Cas20, Corollary 6.8]). However, there is limited detail of the precise connection. Our aim is to find explicit isomorphisms between the various groupoids of filters and groupoids of germs. That is, starting with an arbitrary inverse semigroup with zero, we find an isomorphism $\pi: \mathcal{F} \rightarrow \mathcal{G}_{0}$ from the groupoid $\mathcal{F}$ of proper filters to the groupoid $\mathcal{G}_{0}$ of proper germs (Theorem 3.4.1), and we show how $\pi$ restricts to the subgroupoids of ultrafilters and tight filters.

When the project began, we were mainly interested in inverse semigroups associated to self-similar groups. We emphasise the importance of this special case. It is shown in EP17 that self-similar graphs (which generalise selfsimilar groups) yield a unifying class of $\mathrm{C}^{*}$-algebras, where each $\mathrm{C}^{*}$-algebra is the groupoid $\mathrm{C}^{*}$-algebra of Exel's tight groupoid of germs associated to the inverse semigroup of a given self-similar graph.

In the self-similar group setting, we found an isomorphism between the groupoid of germs and the groupoid of filters. As we put the details together, we realised that our isomorphism would generalise to an arbitrary inverse semigroup with zero, giving rise to $\pi: \mathcal{F} \rightarrow \mathcal{G}_{0}$. This generalisation bridged the project with a prior collaboration between Lisa Orloff Clark, Becky Armstrong and Ying-Fen Lin. Together with Astrid an Huef, our results are available in preprint as Arm+20, which condenses the results of this thesis and omits the self-similar group application.

We will consider a number of structures whose notation is collated here:

- $S$ is a fixed inverse semigroup, as of Assumption 2.1.9.

- $E$ is the set of idempotents in $S$,

- $\mathcal{L}$ is the set of filters in $S$, which will be endowed with a multiplication so that $\mathcal{L}$ is an inverse semigroup, and we get a groupoid $\mathcal{L}$ of filters 
by restricting the multiplication,

- $\mathcal{F}$ is the groupoid of proper filters,

- $\mathrm{F}(E)$ is the set of proper filters in $E$,

- $\mathcal{T}$ is the groupoid of tight filters,

- $\mathcal{U}$ is the groupoid of ultrafilters,

- $\mathcal{G}_{0}$ is the groupoid of proper germs,

- $\mathcal{G}_{\text {tight }}$ is Exel's tight groupoid, and

- $\mathcal{G}_{\infty}$ is the groupoid of ultragerms.

We will revisit the below guiding diagram and highlight what part of the diagram we are establishing in some sections of the thesis.

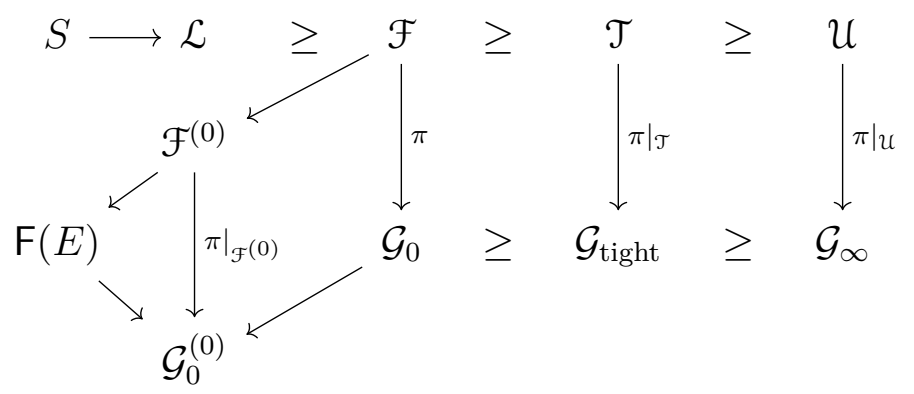

We write $\mathcal{G} \geq \mathcal{H}$ to mean $\mathcal{H}$ is a subgroupoid of $\mathcal{G}$.

We will outline the content of some subsections with this formatting.

A roadmap for the thesis is as follows. In Section 2.1, we give some definitions and fix an inverse semigroup $S$ with zero. We then outline rudiments of the natural partial order on $S$. In Section 2.2, we define filters and a filter composition, which gives the inverse semigroup $\mathcal{L}$ of filters. In Section 3.1. we see groupoids of filters and germs that represent the filter approach 
and germ approach discussed above. Some preliminary interactions between these structures prepare us for the topological isomorphism $\pi: \mathcal{F} \rightarrow \mathcal{G}_{0}$. The class of étale groupoids is defined in Section 3.2. Tools developed while showing a groupoid of filters is étale will support the definition of $\pi: \mathcal{F} \rightarrow \mathcal{G}_{0}$. In Section 3.3, we identify the unit spaces of $\mathcal{F}$ and $\mathcal{G}_{0}$ with $\mathrm{F}(E)$. This identification implies a map between the unit spaces of the groupoids that extends to $\pi: \mathcal{F} \rightarrow \mathcal{G}_{0}$ in Section 3.4. We conclude in Section 3.5 by examining how $\pi$ allows filters in inverse semigroups to be described in terms of the filters of idempotents, which we apply to self-similar groups. 


\section{Chapter 2}

\section{Inverse semigroups}

\subsection{Inverse semigroups}

We define an inverse semigroup $S$ with zero, establishing the first structure in the diagram, and we discuss the natural partial order of the inverse semigroup on which the definitions of filters and germs depend.

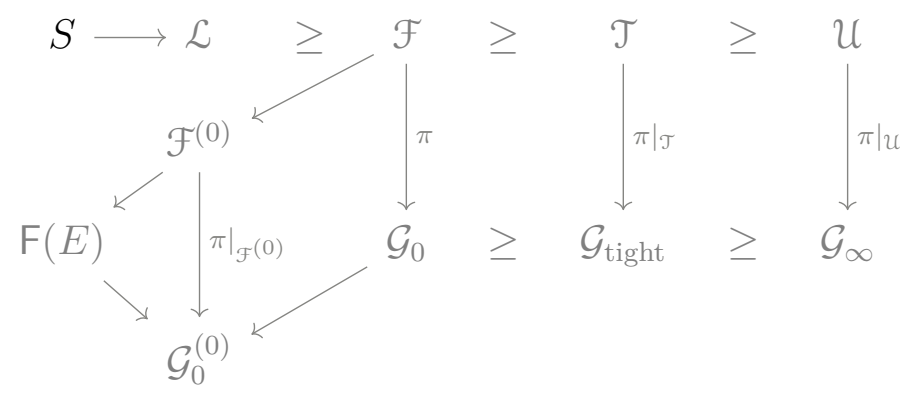

\subsubsection{Preliminaries}

We refer the reader to [How76] and [Law98] for treatments of semigroups and inverse semigroups, respectively. A semigroup $S$ is a set with an associative binary operation. Given $x$ and $y$ in a semigroup $S$, we say $x$ is an idempotent if $x x=x$, and we say $y$ is an inverse of $x$ if

$$
x y x=x \text { and } y x y=y .
$$


We say $S$ is a regular semigroup if every element has an inverse, and $S$ is an inverse semigroup if every element has a unique inverse, in which case the inverse of $x$ is denoted by $x^{*}$.

The above definition has appeared explicitly in the literature as early as How76, §V.1, pp. 129].1. Inverse semigroups were originally defined to be regular semigroups whose idempotents commute, when they were independently introduced by Wagner Vag52 and Preston Pre54a; Pre54b; Pre54c] : $^{2}$ We introduce elementary properties of inverse semigroups toward a proof of Theorem 2.1.7, verifying the definitions are equivalent.

Let $S$ be an inverse semigroup. Denote by $E$ the set of idempotents in $S$. Two properties are immediate from the uniqueness of inverses in $S$.

Lemma 2.1.1. For all $x \in S,\left(x^{*}\right)^{*}=x$.

We say $x$ in $S$ is self-inverse if $x^{*}=x$.

Lemma 2.1.2. Idempotents are self-inverse.

Idempotents are well-behaved in many ways, but we have yet to see that such elements exist. Indeed they do. Define $\mathbf{d}(x):=x^{*} x$, which we call the source of $x$, for each $x \in S$.

Lemma 2.1.3. The set $E$ of idempotents in $S$ is $E=\mathbf{d}(S)$.

Proof. Fix $e \in E$. Then, $e^{*}=e$ by Lemma 2.1.2. Thus,

$$
e=e e=e^{*} e=\mathbf{d}(e) \in \mathbf{d}(S) .
$$

If $x^{*} x \in \mathbf{d}(S)$, then $\left(x^{*} x\right)\left(x^{*} x\right)=x^{*}\left(x x^{*} x\right)=x^{*} x$, so $x^{*} x \in E$.

\footnotetext{
${ }^{1}$ Quoting Pet84 §I.1, pp. 2], "the simple and [a]esthetically pleasing axioms for inverse semigroups have exercised a certain charm upon many researchers in the field of semigroups."

${ }^{2}$ Preston was aware of Wagner's work no later than when he wrote the final note in Pre54c acknowledging the equivalence between their definitions of inverse semigroups. Charles Ehresmann is also due acknowledgement for early work relating to inverse semigroups via inductive groupoids Ehr57, Ehr60.
} 
Knowing idempotents are prevalent via the source map d: $S \rightarrow E$, we investigate the good behaviour of $E$ in more detail.

Lemma 2.1.4. Idempotents are closed under multiplication.

Proof. Fix $e, f \in E$. We show $f(e f)^{*} e$ is an inverse of $e f$, like in Law98, Theorem 1.1.3]. Observe

$$
e f\left(f(e f)^{*} e\right) e f=e(f f)(e f)^{*}(e e) f=e f(e f)^{*} e f=e f
$$

and

$$
f(e f)^{*} e(e f) f(e f)^{*} e=f(e f)^{*}(e e)(f f)(e f)^{*} e=f\left((e f)^{*} e f(e f)^{*}\right) e=f(e f)^{*} e,
$$

so $f(e f)^{*} e$ is an inverse of $e f$. Inverses are unique, so $(e f)^{*}=f(e f)^{*} e$. Further,

$$
\left(f(e f)^{*} e\right)\left(f(e f)^{*} e\right)=f\left((e f)^{*} e f(e f)^{*}\right) e=f(e f)^{*} e \in E .
$$

Hence, $(e f)^{*} \in E$, which means ef $=\left((e f)^{*}\right)^{*}=(e f)^{*} \in E$ by Lemmas 2.1.1 and 2.1.2.

Lemma 2.1.5. The set $E$ is commutative.

Proof. Fix $e, f \in E$. Because $E$ is closed under multiplication (Lemma 2.1.4), $e f, f e \in E$, and idempotents are self-inverse (Lemma 2.1.2), so $(e f)^{*}=e f$. Since inverses are unique, it suffices to show $f e$ is an inverse of $e f$. Observe that

$$
\text { effeef }=e f e f=e f \text { and } f e e f f e=f e f e=f e
$$

because $e, f, e f, f e \in E$, so $f e$ is an inverse of $e f$.

Now we can show the operations in $S$ cooperate in the following sense.

Lemma 2.1.6. For all $x, y \in S,(x y)^{*}=y^{*} x^{*}$. 
Proof. Idempotents commuting (Lemma 2.1.5) and $E=\mathbf{d}(S)$ (Lemma 2.1.3) imply

$$
x y\left(y^{*} x^{*}\right) x y=x\left(y y^{*}\right)\left(x^{*} x\right) y=x x^{*} x y y^{*} y=x y
$$

and

$$
y^{*} x^{*} x y y^{*} x^{*}=y^{*} y y^{*} x^{*} x x^{*}=y^{*} x^{*},
$$

so $y^{*} x^{*}$ is an inverse of $x y$. Inverses are unique, so $(x y)^{*}=y^{*} x^{*}$.

The following characterisation of inverse semigroups was proved independently by Liber, and by Munn and Penrose together. We cite Wagner's presentation of the result in Vag53, Theorem 3.3].

Theorem 2.1.7. Let $S$ be a semigroup. The following are equivalent:

(1) $S$ is a regular semigroup whose idempotents commute;

(2) $S$ is an inverse semigroup.

Proof. Lemma 2.1.5 tells us that (2) implies (1). Suppose (1) holds. Fix $x \in S$. Since $S$ is regular, $x$ has at least one inverse. Suppose $a$ and $b$ are inverses of $x$. Then, $x a x=x, a x a=a, x b x=x$ and $b x b=b$. Observe $(x a)(x a)=(x a x) a=x a$, so $x a \in E$. Similarly, we can show $a x, x b, b x \in E$. Notice

$$
a=a x a=a(x b x) a=(a x)(b x) a=(b x)(a x) a=b x a,
$$

and

$$
b x a=b(x b x) a=b(x b)(x a)=b(x a)(x b)=b x b=b,
$$

since idempotents commute. Hence, $a=b$.

Example 2.1.8 (Proposition 1.1.2 of Law98). Let $X$ be a set. A partial function on $X$ is a function $f: A \rightarrow B$, where $A, B \subseteq X$. A bijective partial function is called a partial bijection on $X$. The set of partial bijections on $X$ is denoted by $\mathcal{J}(X)$. The composition of two partial functions is the usual composition but defined on the largest domain possible. Under partial function composition $\mathfrak{J}(X)$ is an inverse semigroup. 
Assumption 2.1.9. Let $S$ be an inverse semigroup.

Observe that the set $E$ of idempotents in $S$ is an inverse semigroup because $E$ is closed under multiplication and inversion by Lemmas 2.1.4 and 2.1 .2 , respectively.

Note that the tools established in this section will be used without reference throughout the thesis.

\subsubsection{Assuming zero exists}

A zero in $S$ is an element, denoted by 0 , such that $x 0=0 x=0$, for all $x \in S$. If there is a zero in $S$, then we say $S$ is an inverse semigroup with zero, and any $x$ in $S$ such that $x \neq 0$ is nonzero.

Zeros can be troublesome. A group is an inverse semigroup with a unique idempotent Law98, Proposition 1.4.4, pp. 19]. The real numbers with the standard multiplication is not a group because both zero and one would be idempotents. (In general, a group contains a zero if, and only if, the group is trivial.) However, the reals under multiplication is an inverse semigroup whose idempotents are zero and one, so there are nontrivial inverse semigroups with zero. Better yet, we will see that, if $S$ contains a zero, then the zero is the minimum element of $S$ with respect to the natural partial order. Moreover, any inverse semigroup can be embedded in an inverse semigroup with zero by adding a new element, denoted by 0 , and extending the multiplication appropriately. Thus, it is both helpful and reasonable to restrict our attention to inverse semigroups with zero, like in [Exe09].

Assumption 2.1.10. Assume $S$ is an inverse semigroup with zero ${ }^{3}$

Notice that $E$ is an inverse semigroup with zero because $0 \in E$.

\footnotetext{
${ }^{3}$ Quoting Exe09], "one may wonder why in the world would anyone want to insert a zero in an otherwise well behaved semigroup. Rather than shy away from inverse semigroups with zero, we will assume that all of them contain a zero element, not least because we want to keep a close eye on this exceptional element".
} 


\subsubsection{Natural partial order}

We define a partial order on $S$ that is essential to the definition of both filters and germs. The results of the present section are in Law98.

A partial order on a set is a binary relation that is reflexive, antisymmetric and transitive. The natural partial order on $S$ is the relation

$$
a \leq b \Longleftrightarrow a=e b \text { for some } e \in E \text {. }
$$

Lemma 2.1.11. The natural partial order is a partial order.

Proof. The relation $\leq$ is reflexive because $\mathbf{d}(S)=E$ and $a=a a^{*} a$, for all $a \in S$. Transitivity follows from $E$ being closed under multiplication. Lastly, $\leq$ is antisymmetric because idempotents commute.

Lemma 2.1.12. For all $a, b \in S$, the following are equivalent:

(1) $a \leq b$,

(2) $a=a a^{*} b$,

(3) $a=b a^{*} a$,

(4) $a=$ be for some $e \in E$, and

(5) $a^{*} \leq b^{*}$.

Proof. We show

$$
(1) \Longrightarrow(2) \Longrightarrow(3) \Longrightarrow(4) \Longrightarrow(1) \Longleftrightarrow(5) \text {. }
$$

Suppose (1), so $a=e b$ for some $e \in E$. Then,

$$
a a^{*} b=e b(e b)^{*} b=e b b^{*} e^{*} b=e e^{*} b b^{*} b=e e b=e b=a,
$$

so (2) holds. If (2) holds, then

$$
b a^{*} a=b\left(a a^{*} b\right)^{*} a a^{*} b=b b^{*} a a^{*} a a^{*} b=a a^{*} a a^{*} b b^{*} b=a a^{*} b=a,
$$


so (3) holds. Since $a^{*} a \in E$, if (3) holds, then (4) holds. If (4) holds, then

$$
a a^{*} b=b e(b e)^{*} b=b e b^{*} b=b b^{*} b e=b e=a,
$$

and $a a^{*} \in E$, so (1) holds. Thus,

$$
(1) \Longrightarrow(2) \Longrightarrow(3) \Longrightarrow(4) \Longrightarrow(1) \text {. }
$$

To see $(1) \Longleftrightarrow(5)$. it suffices to show (5) implies $(1)$ because $\left(x^{*}\right)^{*}=x$, for all $x \in S$. If (5) holds, then $x^{*}=e y^{*}$ for some $e \in E$. Then,

$$
x=\left(x^{*}\right)^{*}=\left(e y^{*}\right)^{*}=\left(y^{*}\right)^{*} e^{*}=y e,
$$

so $x=y e$, and it follows that $x \leq y$ because (4) is equivalent to (1).

Lemma 2.1.13. For all $a, b, c, d \in S$, if $a \leq b$ and $c \leq d$, then $a c \leq b d$.

Proof. Suppose $a \leq b$ and $c \leq d$, so $a=b e$ and $c=f d$ for some $e, f \in E$. Observe

$$
a c(a c)^{*} b d=b e f d(b e f d)^{*} b d=b e f d d^{*} e f b^{*} b d=b b^{*} \text { befef } d d^{*} d=b e f d=a c,
$$

so $a c \leq b d$ by Lemma 2.1.12).

We will say the natural partial order in $S$ is compatible with multiplication and inversion in reference to Lemma 2.1.12) and Lemma 2.1.13. Note also that, for all $x, y \in S$, we write $x \geq y$ if, and only if, $y \leq x$.

In the following lemma, we collate other useful facts about $S$.

Lemma 2.1.14. For all $a, b \in S$ and for all $e \in E$,

(1) $0 \leq a$,

(2) $(a b)^{*} a b \leq b^{*} b$,

(3) $a^{*} e a \in E$,

(4) $e a, a e \leq a$, and 
(5) $a \leq e$ implies $a \in E$.

Proof. Fix $a, b \in S$ and let $e \in E$.

(1) Notice $0=0 a$ and $0 \in E$, so $0 \leq a$ by definition.

(2) Observe

$(a b)^{*} a b\left((a b)^{*} a b\right)^{*} b^{*} b=(a b)^{*} a b(a b)^{*} a b b^{*} b=(a b)^{*} a b(a b)^{*} a b=(a b)^{*} a b$.

(3) Notice $a^{*} e a a^{*} e a=a^{*} a a^{*} e e a=a^{*} e a$.

(4) Compute $e a(e a)^{*} a=e a a^{*} e^{*} a=e e^{*} a a^{*} a=e a$, so $e a \leq a$, and a similar computation shows $a e \leq a$.

(5) Suppose $a \leq e$. Then, $a=f e$ for some $f \in E$, and $E$ is closed under multiplication (Lemma 2.1.4), so $a \in E$.

For all $s, t \in S$, if $\{s, t\}$ has a greatest lower bound $u$ with respect to the natural partial order, we say $u$ is the meet of $s$ and $t$, and we write $u=s \wedge t$.

Lemma 2.1.15. For all $e, f \in E, e \wedge f$ exists and equals ef.

Proof. Notice $e f=(e f) e=(e f) f$, so $e f$ is a lower bound of $\{e, f\}$. Suppose $p$ is a lower bound of $\{e, f\}$. We know $p \in E$ by Lemma 2.1.14)(5), so

$$
p=p p \leq e f
$$

because the natural partial order is compatible with multiplication (Lemma 2.1.13). Thus, ef is the greatest lower bound of $\{e, f\}$.

\section{$2.2 \quad$ Filters}

We define filters $\mathcal{L}$, proper filters $\mathcal{F}$, ultrafilters $\mathcal{U}$, idempotent proper filters $\mathcal{F}^{(0)}$ and proper filters of idempotents $\mathrm{F}(E)$. A composition of filters yields the inverse semigroup $\mathcal{L}$ of filters, and we show $S$ embeds in $\mathcal{L}$ as in the guiding diagram below. 


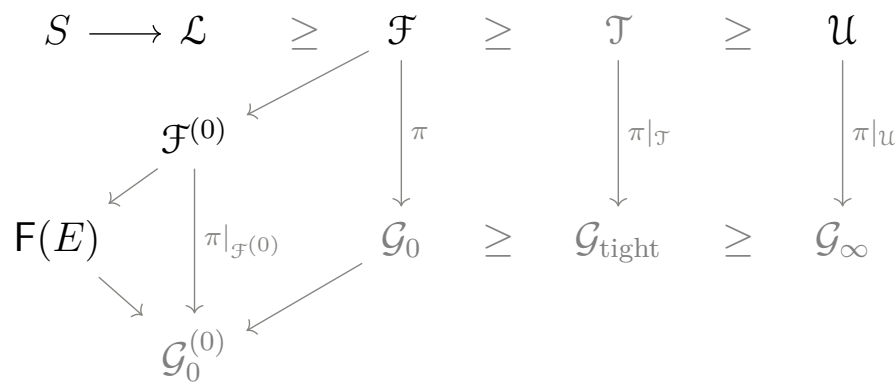

We use similar notation and terminology to Law10b for our description of filters. Given $A \subseteq S$, we write

$$
A^{\uparrow}:=\{b \in S: \exists a \in A(a \leq b)\} \text { and } A^{\downarrow}:=\{b \in S: \exists a \in A(b \leq a)\}
$$

Also,

$$
a^{\uparrow}:=\{a\}^{\uparrow} \text { and } a^{\downarrow}:=\{a\}^{\downarrow},
$$

for all $a \in S$. To exercise the new notation, observe $0^{\uparrow}=S, 0^{\downarrow}=\{0\}$ and $E^{\downarrow}=E$ from Lemma 2.1.14. The reflexivity and transitivity of the natural partial order implies $\left(A^{\uparrow}\right)^{\uparrow}=A^{\uparrow}$. We say $A$ is down-directed if, for all $a, b \in A$, there is $c \in A$ such that $c \leq a, b$. We say $A$ is an up-set if $A^{\uparrow}=A$. Given an up-set $A$, an element $a \in A$ and $b \in S$ such that $a \leq b$, we will often form our arguments as follows. Notice

$$
A \ni a \leq b
$$

which implies $b \in A$ because $A$ is an up-set. A filter is a nonempty downdirected up-set in $S$. We denote the set of filters in $S$ by $\mathcal{L}$.

\subsubsection{Trivial and proper filters}

Since $S$ has a zero, the subset $S$ of the inverse semigroup $S$ is a filter in $S$, which we call the trivial filter. Some authors assume filters are proper subsets, like in [LL13, Remark 1.1]. In Section 2.2.4, we show the inverse semigroup $S$ embeds in an inverse semigroup of filters in which the trivial 
filter is the zero. Thus, permitting the trivial filter reflects the zero in the inverse semigroup $S$ from Assumption 2.1.10.

That said, we will work primarily with filters that are not trivial, which we call proper filters. Since 0 is the minimum in $S$, a filter is proper if, and only if, it does not contain 0 . We denote the set of proper filters in $S$ by $\mathcal{F}$.

\subsubsection{Ultrafilters}

A filter in $S$ that is maximal among proper filters is an ultrafilter. That is, a proper filter $F$ in $S$ is an ultrafilter if, and only if,

$$
G \supseteq F \text { implies } G=S \text { or } G=F
$$

for all $G \in \mathcal{L}$. We denote the set of ultrafilters in $S$ by $\mathcal{U}$. Filters are abundant because $x^{\uparrow}$ is a filter, for all $x \in S$. For the existence of ultrafilters, we assume Zorn's lemma.

Given a partial order $\leq$ on a set $P$, a chain is a subset of $P$ that is antisymmetric, transitive and satisfies that either $x \leq y$ or $y \leq x$, for all $x, y \in P$. That is, a chain is a totally ordered subset of $P$. We state Zorn's lemma as in Hal60, §16, pp. 62].

Theorem 2.2.1 (Zorn's lemma). Let $P$ be a partially ordered set. If every chain in $P$ has an upper bound, then $P$ contains a maximal element.

Corollary 2.2.2. Every proper filter is contained in an ultrafilter.

Proof. We give the details of a standard argument using Zorn's lemma appearing on UT18. The power set of $S$ is partially ordered by the inclusion relation. We show the union of a chain of proper filters is a proper filter. Let $\left(F_{k}\right)_{k \in K}$ be a chain in the power set of $S$, and put $G:=\bigcup_{k \in K} F_{k}$. Every $F_{k}$ is nonempty, so $G$ is nonempty. Fix $a, b \in G$. Find $j, k \in K$ so that $a \in F_{j}$ and $b \in G_{k}$. Either $F_{j} \subseteq F_{k}$ or $F_{k} \subseteq F_{j}$ because $\left(F_{k}\right)_{k \in K}$ is a chain. We suppose without loss of generality that $F_{j} \subseteq F_{k}$. Then, $a, b \in F_{k}$, and $F_{k}$ is down-directed, so there is $c \in F_{k} \subseteq G$ such that $c \leq a, b$. Hence, $G$ is 
down-directed. Fix $c \in S$ and suppose $a \leq c$. Then, $c \in F_{j} \subseteq G$ because $F_{j}$ is an up-set. Thus, $G$ is an up-set. Lastly, notice every $F_{k}$ is proper, so $G$ is a proper filter.

We show every proper filter is contained in an ultrafilter by Zorn's lemma. Fix a proper filter $F$. Let $\mathcal{F}_{F}:=\{G \in \mathcal{F}: F \subseteq G\}$. The set $\mathcal{F}_{F}$ is partially ordered by inclusion. We show every chain in $\mathcal{F}_{F}$ has an upper bound. If there are no chains in $\mathcal{F}_{F}$, then the claim holds vacuously. Otherwise, let $\left(G_{k}\right)_{k \in K}$ be a chain in $\mathcal{F}_{F}$. By the above argument, $\bigcup_{k \in K} G_{k}$ is a proper filter. Also, $F \subseteq G_{j} \subseteq \bigcup_{k \in K} G_{k}$, for all $j \in K$, so $\bigcup_{k \in K} G_{k} \in \mathcal{F}_{F}$ and $\bigcup_{k \in K} G_{k}$ is an upper bound on the chain $\left(G_{k}\right)_{k \in K}$. Thus, every chain in $\left(G_{k}\right)_{k \in K}$ has an upper bound, so Zorn's lemma (Theorem 2.2.1) implies $\mathcal{F}_{F}$ contains a maximal element $U$. Fix $G \in \mathcal{F}$. Suppose $U \subseteq G$. Then, $F \subseteq G$ because $F \subseteq U$, so $G \in \mathcal{F}_{F}$, and $U$ is maximal in $\mathcal{F}_{F}$, so $U=G$. Hence, $U$ is an ultrafilter containing $F$.

\subsubsection{Idempotent filters and filters of idempotents}

Recall $E$ is the set of idempotents in $S$. Given $F \in \mathcal{L}$, we say $F$ is idempotent if $F \cap E \neq \emptyset$. The terminology is reinforced by Proposition 2.2 .8 where we show $\mathcal{L}$ is an inverse semigroup whose idempotents are precisely the idempotent filters. We denote the set of idempotent proper filters by $\mathcal{F}^{(0)}$ and the set of idempotent ultrafilters by $\mathcal{U}^{(0)}$. The notation will be explained in Chapter 3, where we see that the groupoid $\mathcal{F}$ of proper filters and the groupoid $\mathcal{U}$ of ultrafilters have unit spaces $\mathcal{F}^{(0)}$ and $\mathcal{U}^{(0)}$, respectively.

Recall from Section 2.1 .2 that $E$ is an inverse semigroup with zero, so there are filters in $E$. We denote the set of proper filters in $E$ by $\mathrm{F}(E)$ and the set of ultrafilters in $E$ by $\mathrm{U}(E)$. 


\subsubsection{Inverse semigroup of filters}

We show $S$ embeds in an inverse semigroup $\mathcal{L}$ of filters. We characterise reverse inclusion of filters as the natural partial order on $\mathcal{L}$ in order to define the groupoid $\mathcal{U}$ of ultrafilters.

Lemma 2.2.3. For all $A, B \subseteq S$, and for all $x \in S$, if $A$ and $B$ are downdirected, then $A B, x B$ and $A x$ are down-directed.

Proof. Let $A, B \subseteq S$. Fix $a b, c d \in A B$ so that $a, c \in A$ and $b, d \in B$. Because $A$ and $B$ are down-directed, there are $x \in A$ and $y \in B$ such that $x \leq a, c$ and $y \leq b, d$. Since the natural partial order is compatible with multiplication (Lemma 2.1.13), $x y \leq a b, c d$, and $x y \in A B$. Therefore, $A B$ is down-directed. The reflexivity of the natural partial order implies singletons are down-directed, so the remainder of the lemma is a special case.

Lemma 2.2.4. If $A \subseteq S$ is nonempty and down-directed, then $A^{\uparrow}$ is a filter.

Proof. This follows from $\left(A^{\uparrow}\right)^{\uparrow}=A^{\uparrow}$, for all $A \subseteq S$.

Any two filters $F$ and $G$ are down-directed, so Lemma 2.2 .3 implies $F G$ is down-directed. Thus, $(F G)^{\uparrow}$ is a filter by Lemma 2.2.4, so we have a map

$$
(F, G) \mapsto F \cdot G:=(F G)^{\uparrow}
$$

from $\mathcal{L} \times \mathcal{L}$ to $\mathcal{L}$ as in Law10b], called filter composition. The associativity of the multiplication in $S$ ensures filter composition in $\mathcal{L}$ is associative, so $\mathcal{L}$ is a semigroup. It is known that $\mathcal{L}$ is an inverse semigroup [LMS13, §3.1]. We provide a proof for completeness.

For any $A \subseteq S$, we write $A^{*}:=\left\{a^{*}: a \in A\right\}$.

Lemma 2.2.5 (Lemma 2.6 of Law10b]). For all $F \in \mathcal{L}, F=F F^{*} F$.

Proof. Let $F \in \mathcal{L}$. For all $f \in F, f=f f^{*} f$, so we have $F \subseteq F F^{*} F$. Fix $f g^{*} h \in F F^{*} F$, where $f, g, h \in F$. Since filters are down-directed, there is 
$a \in F$ such that $a \leq f, g, h$. Then,

$$
F \ni a=a a^{*} a \leq f g^{*} h,
$$

which implies $f g^{*} h \in F$ because filters are up-sets. Hence, $F \supseteq F F^{*} F$.

Lemma 2.2.6. The semigroup $\mathcal{L}$ is an inverse semigroup.

Proof. Fix $F \in \mathcal{L}$. Lemma 2.2 .5 implies $F=F F^{*} F \subseteq F \cdot F^{*} \cdot F$. Fix $x \in F \cdot F^{*} \cdot F$. There exists $f, g, h \in F$ such that $f g^{*} h \leq x$, using transitivity. Filters are down-directed, so there is $a \in F$ such that $a \leq f, g, h$. Then,

$$
F \ni a=a a^{*} a \leq f g^{*} h \leq x
$$

which implies $x \in F$. Therefore, $F \subseteq F \cdot F^{*} \cdot F \subseteq F$, and so $F=F \cdot F^{*} \cdot F$. Notice $\left(F^{*}\right)^{*}=F$, so $F^{*}=F^{*} \cdot F \cdot F^{*}$ follows from a similar argument. Therefore, $F^{*}$ is an inverse of $F$.

We show $F^{*}$ is the unique inverse of $F$. Suppose also $G$ is an inverse of $F$. We show $G=F^{*}$. Fix $g \in G$. Filters are nonempty, so take $f \in F$. Observe

$$
g \geq f^{*} f g f f^{*} \in F^{*} \cdot F \cdot G \cdot F \cdot F^{*}=F^{*} \cdot F \cdot F^{*}=F^{*}
$$

because $G$ is an inverse of $F$, and so $g \in F^{*}$. Thus, $G \subseteq F^{*}$.

Because $G \cdot F \cdot G=G$, we know $G^{*} \cdot F^{*} \cdot G^{*}=G^{*}$. Then, it follows from a similar argument to above that $G \supseteq F^{*}$.

Thus, the inverse semigroup $S$ yields the inverse semigroup $\mathcal{L}$ of filters, which is denoted by $L(S)$ in Law10b, Proposition 2.12]. Indeed the inverse semigroups are closely related as follows. A map $\phi$ from $S$ to an inverse semigroup $T$ is called a homomorphism if $\phi(x y)=\phi(x) \phi(y)$, for all $x, y \in S$. An injective homomorphism $\phi: S \rightarrow T$ is called an embedding. Proposition 2.2.7 below is known LMS13, §3.1, pp. 245], but we provide another proof for completeness.

Proposition 2.2.7. The map $x \mapsto x^{\uparrow}$ from $S$ to $\mathcal{L}$ is an embedding. 
Proof. Fix $a, b \in S$. Compatibility of the natural partial order with multiplication implies $a^{\uparrow} \cdot b^{\uparrow} \subseteq(a b)^{\uparrow}$, and $a^{\uparrow} \cdot b^{\uparrow} \supseteq(a b)^{\uparrow}$ holds because $a b \in a^{\uparrow} \cdot b^{\uparrow}$. Thus, $a^{\uparrow} \cdot b^{\uparrow}=(a b)^{\uparrow}$. Suppose $a^{\uparrow}=b^{\uparrow}$. Reflexivity of $\leq$ implies

$$
a \in a^{\uparrow}=b^{\uparrow} \ni b,
$$

so $a \in b^{\uparrow}$ and $b \in a^{\uparrow}$. Then, antisymmetry of $\leq$ implies $a=b$.

We aim to answer three questions about the inverse semigroup $\mathcal{L}$. What are its idempotents? What is the natural partial order? Does it have a zero?

Recall that a filter in $S$ is idempotent if it intersects $E$.

Lemma 2.2.8 (Lemma 2.8(2) of [Law12]). The idempotents in the inverse semigroup $\mathcal{L}$ are precisely the idempotent filters.

Proof. Suppose $F$ is an idempotent in the inverse semigroup $\mathcal{L}$. Filters are nonempty, so take $x \in F$. Idempotents are self-inverse, so

$$
x^{*} x \in F^{*} F \subseteq F^{*} \cdot F=F \cdot F=F,
$$

and $x^{*} x \in E$. Hence, $F$ is an idempotent filter.

Now suppose $F$ is an idempotent filter. Find $e \in F \cap E$. Fix $x \in F$. Since $e x \in F F \subseteq F \cdot F$, we have

$$
F \cdot F \ni e x \leq x
$$

which implies $x \in F \cdot F$ because filters are up-sets. Now fix $y \in F \cdot F$, and find $a, b \in F$ so that $a b \leq y$. Filters are down-directed, so there is $c \in F$ such that $c \leq a, b$, in which case $c c \leq y$. Using down-directedness for a second time, we find $d \in F$ so that $d \leq c, e$. Then, $d d \leq y$, but $d$ is an idempotent because $d \in e^{\downarrow} \subseteq E^{\downarrow}=E$, so $d=d d \leq y$. Hence,

$$
F \ni d \leq y
$$

which implies $y \in F$. Therefore, $F \cdot F=F$, and so $F$ is an idempotent in the inverse semigroup $\mathcal{L}$. 
Lemma 2.2.9 (§3.1 of LMS13]). The natural partial order $\leq_{\mathcal{L}}$ on the inverse semigroup $\mathcal{L}$ of filters is reverse inlcusion. That is,

$$
F \supseteq G \Longleftrightarrow F \leq_{\mathcal{L}} G,
$$

for all $F, G \in \mathcal{L}$.

Proof. Suppose $F \supseteq G$. We claim $F \cdot F^{*} \cdot G=F$. Observe

$$
F \cdot F^{*} \cdot G \subseteq F \cdot F^{*} \cdot F=F .
$$

It remains to show $F \subseteq F \cdot F^{*} \cdot G$. Fix $f \in F$. Filters are nonempty, so take $g \in G$. Then, $g \in F$ because $G \subseteq F$. Filters are down-directed, so there is $h \in F$ such that $h \leq f, g$. Then,

$$
F \cdot F^{*} \cdot G \ni h h^{*} g=h \leq f
$$

which implies $f \in F \cdot F^{*} \cdot G$. Hence, $F=F \cdot F^{*} \cdot G$, so $F \leq_{\mathcal{L}} G$.

Now suppose $F \leq_{\mathcal{L}} G$. That is, $F=F \cdot F^{*} \cdot G$. Fix $g \in G$. Filters are nonempty, so take $f \in F$. Because $f f^{*} \in E$, we have $f f^{*} g \leq g$. Then,

$$
F=F \cdot F^{*} \cdot G \ni f f^{*} g \leq g,
$$

which implies $g \in F$. Therefore, $F \supseteq G$.

The characterisation of the natural partial order on $\mathcal{L}$ in Lemma 2.2.9 will help us to construct the groupoid $\mathcal{U}$ of ultrafilters in Section 3.1 .2 .

Lemma 2.2.10. The trivial filter $S$ is the zero in the inverse semigroup $\mathcal{L}$.

Proof. For any $F \in \mathcal{L}, F \cdot S=(F S)^{\uparrow}=S=(S F)^{\uparrow}=S \cdot F$.

Recall from Section 2.1.1 that inverse semigroups have source maps. In particular, for each $F \in \mathcal{L}$, we have $\mathbf{d}(F)=F^{*} \cdot F$. Any filter can be reconstructed from any member of and the source of the filter as per the following lemma, which is Proposition 1.4 of Law93. 
Lemma 2.2.11. For all $F \in \mathcal{L}$ and for all $x \in F$,

$$
F=(x \mathbf{d}(F))^{\uparrow} .
$$

Proof. Fix $F \in \mathcal{L}$ and $x \in F$. Using the definition of filter composition,

$$
(x \mathbf{d}(F))^{\uparrow} \subseteq(F \mathbf{d}(F))^{\uparrow}=F \cdot \mathbf{d}(F)=F .
$$

Fix $f \in F$. Filters are down-directed, so there is $y \in F$ such that both $y \leq x$ and $y \leq f$. The former means $y=x y^{*} y$. Together with the latter, we get $x y^{*} y \leq f$. That is, $f \in\left(x y^{*} y\right)^{\uparrow} \subseteq(x \mathbf{d}(F))^{\uparrow}$. Hence, $F \subseteq(x \mathbf{d}(F))^{\uparrow}$.

This way of reconstructing filters will be critical in Theorem 3.4.1, where we show there is a topological isomorphism $\pi: \mathcal{F} \rightarrow \mathcal{G}_{0}$. 


\section{Chapter 3}

\section{Groupoids}

\subsection{Groupoids of filters and germs}

We define groupoids of filters and germs and observe important interactions that prepare us for the topological isomorphism $\pi: \mathcal{F} \rightarrow \mathcal{G}_{0}$. In terms of our guiding diagram, we establish the groupoid $\mathcal{F}$ of proper filters and the groupoid $\mathcal{G}_{0}$ of proper germs, as well as their unit spaces $\mathcal{F}^{(0)}$ and $\mathcal{G}_{0}^{(0)}$.

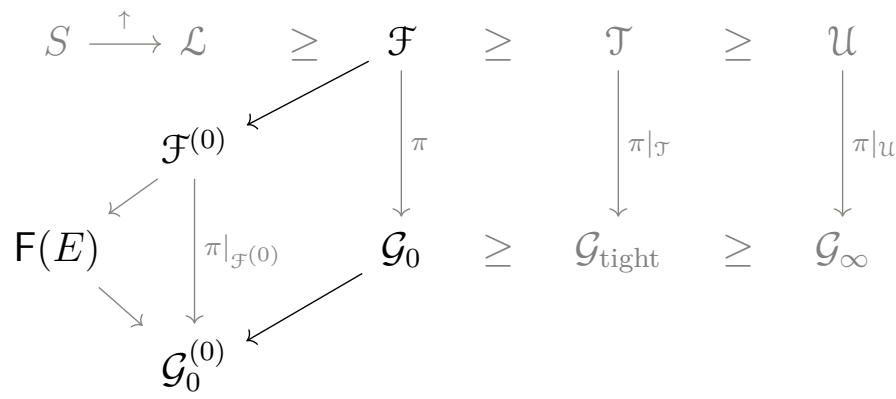

\subsubsection{Groupoids associated to inverse semigroups}

We give the rudiments of groupoids as in [Sim18], and we consider our first notion of a groupoid associated to an inverse semigroup. 
A groupoid ${ }^{1}$ is a set $\mathcal{G}$ with a subset $\mathcal{G}^{(2)} \subseteq \mathcal{G} \times \mathcal{G}$, a composition $(\alpha, \beta) \mapsto$ $\alpha \beta$ from $\mathcal{G}^{(2)}$ to $\mathcal{G}$ and an inversion $\gamma \mapsto \gamma^{-1}$ on $\mathcal{G}$ so that, for all $\alpha, \beta, \gamma \in \mathcal{G}$,

(1) $\left(\gamma^{-1}\right)^{-1}=\gamma$ and $\left(\gamma^{-1}, \gamma\right) \in \mathcal{G}^{(2)}$,

(2) if $(\alpha, \beta),(\beta, \gamma) \in \mathcal{G}^{(2)}$, then $(\alpha, \beta \gamma),(\alpha \beta, \gamma) \in \mathcal{G}^{(2)}$ and $\alpha(\beta \gamma)=(\alpha \beta) \gamma$, and

(3) if $(\gamma, \eta) \in \mathcal{G}^{(2)}$, then $\gamma^{-1} \gamma \eta=\eta$ and $\gamma \eta \eta^{-1}=\gamma$.

Given a groupoid $\mathcal{G}$, the elements of the subset $\mathcal{G}^{(2)}$ are called the composable pairs. The units are the elements of the unit space

$$
\mathcal{G}^{(0)}:=\left\{\gamma^{-1} \gamma: \gamma \in \mathcal{G}\right\}
$$

The source $\mathbf{d}$ and range $\mathbf{r}$ in $\mathcal{G}$ are maps from $\mathcal{G}$ to $\mathcal{G}$ defined by $\mathbf{d}(\gamma):=\gamma^{-1} \gamma$ and $\mathbf{r}(\gamma):=\gamma \gamma^{-1}$. A subset $\mathcal{H}$ of a groupoid $\mathcal{G}$ is a subgroupoid if, for all $\alpha, \beta \in \mathcal{H}, \alpha^{-1} \in \mathcal{H}$ and $(\alpha, \beta) \in \mathcal{G}^{(2)}$ implies $\alpha \beta \in \mathcal{H}$. A subgroupoid is a groupoid.

Inverse semigroups and groupoids both generalise groups. The notable difference is that groupoid composition is partial while inverse semigroup multiplication is total, so it is natural to ask if we can restrict the inverse semigroup product to get a groupoid. Notice both structures have a totally defined inversion. This common ground indicates how to restrict the product because the partiality of groupoid composition depends on the common ground in the following way.

Lemma 3.1.1 (Lemma 2.1.4 of [Sim18]). Let $\mathcal{G}$ be a groupoid. The composable pairs are given by

$$
\mathcal{G}^{(2)}=\{(\alpha, \beta) \in \mathcal{G} \times \mathcal{G}: \mathbf{d}(\alpha)=\mathbf{r}(\beta)\} .
$$

\footnotetext{
${ }^{1}$ The generalisation of groups to groupoids is due to Brandt, which is documented in his 1927 paper 'On a generalisation of the group' Bra27. The definition we give can be found in Hah78, Definition 1.1], attributed to a suggestion by George Mackey in conversation.
} 
Proof. Suppose $(\alpha, \beta) \in \mathcal{G}^{(2)}$. Notice

$$
\mathbf{d}(\alpha)=\alpha^{-1} \alpha=\left(\left(\alpha^{-1} \alpha\right) \beta\right) \beta^{-1}=\alpha^{-1}\left(\alpha\left(\beta \beta^{-1}\right)\right)=\beta \beta^{-1}=\mathbf{r}(\beta) .
$$

For the reverse inclusion, suppose $(\alpha, \beta) \in \mathcal{G} \times \mathcal{G}$ and $\mathbf{d}(\alpha)=\mathbf{r}(\beta)$. We know $(\alpha, \mathbf{d}(\alpha)) \in \mathcal{G}^{(2)}$, so $(\alpha, \mathbf{r}(\beta)) \in \mathcal{G}^{(2)}$. Also, $(\mathbf{r}(\beta), \beta) \in \mathcal{G}^{(2)}$. Since $(\alpha, \mathbf{r}(\beta)),(\mathbf{r}(\beta), \beta) \in \mathcal{G}^{(2)}$, it follows that $(\alpha, \beta) \in \mathcal{G}^{(2)}$.

Thus, the groupoid composition depends on the common ground between groupoids and inverse semigroups in a precise way, motivating the restricted product below.

Like $\mathbf{d}: S \rightarrow E$, define the range map from $S$ to $E$ by $x \mapsto \mathbf{r}(x):=x x^{*}$. The restricted product in the inverse semigroup $S$ is the restriction of the product in $S$ to

$$
S^{(2)}:=\{(s, t) \in S \times S: \mathbf{d}(s)=\mathbf{r}(t)\} .
$$

The set $S$ endowed with the restricted product and the inversion in $S$ is a groupoid [Law98, Proposition 3.1.4], called the associated groupoid of $S$. The unit space of the associated groupoid of $S$ is $E$ [Pat99, Proposition 1.0.1].

\subsubsection{Groupoids of filters}

We utilise associated groupoids of inverse semigroups to define the groupoid $\mathcal{L}$ of filters, and we extract the groupoid $\mathcal{F}$ of proper filters. To construct the groupoid $\mathcal{U}$ of ultrafilters, we leverage our characterisation of the natural partial order $\leq_{\mathcal{L}}$ on $\mathcal{L}$ to view ultrafilters as 'primitive' elements of $\mathcal{L}$, as in Law10b, §2].

The associated groupoid of the inverse semigroup $\mathcal{L}$ of filters is called the groupoid of filters. Recall a filter in $S$ is idempotent if it contains an idempotent. In Lemma 2.2.8, we saw that the idempotents in the inverse semigroup $\mathcal{L}$ of filters are precisely the idempotent filters, so the unit space $\mathcal{L}^{(0)}$ of the 
groupoid $\mathcal{L}$ of filters is the set of idempotent filters. The composable pairs are

$$
\mathcal{L}^{(2)}=\{(F, G) \in \mathcal{L} \times \mathcal{L}: \mathbf{d}(F)=\mathbf{r}(G)\}
$$

Recall $\mathcal{F}$ denotes the set of proper filters in $S$. In the following lemma, we show $\mathcal{F}$ is a subgroupoid of $\mathcal{L}$, which we call the groupoid of proper filters.

Proposition 3.1.2. The subset $\mathcal{F}$ of the groupoid $\mathcal{L}$ of filters is a subgroupoid.

Proof. Fix $F, G \in \mathcal{F}$. Because the natural partial order is compatible with inversion (Lemma 2.1.14](5)], we know $F^{*} \in \mathcal{F}$. Suppose $(F, G) \in \mathcal{L}^{(2)}$, so $\mathbf{d}(F)=\mathbf{r}(G)$. Assume for a contradiction that $0 \in F \cdot G$. Filters are nonempty, so take $g \in G$. Then,

$$
0=0 g^{*} \in F \cdot G \cdot G^{*}=F \cdot \mathbf{r}(G)=F \cdot \mathbf{d}(F)=F,
$$

but $F$ is proper, so we have a contradiction. Thus, $F \cdot G$ is in $\mathcal{F}$.

Recall that an ultrafilter is a maximal proper filter and that $\mathcal{U}$ denotes the set of ultrafilters in $S$. We use 'primitives' in inverse semigroups to characterise the groupoid $\mathcal{U}$ of ultrafilters, as per Law10b, §2]. A nonzero element $a$ in $S$ is said to be primitive if

$$
b \leq a \text { implies } b=0 \text { or } b=a
$$

as per Law98, 9.2, pp. 278], and

$$
\operatorname{Prim}(S):=\{a \in S: a \text { is primitive }\} \cup\{0\}
$$

Recall from Lemma 2.2 .10 that $\mathcal{L}$ is an inverse semigroup with zero $S$, so $\operatorname{Prim}(\mathcal{L})$ is defined.

Lemma 3.1.3. The set $\operatorname{Prim}(\mathcal{L})$ is an inverse semigroup, and

$$
\operatorname{Prim}(\mathcal{L})=\mathcal{U} \cup\{S\}
$$


Proof. Consider the inverse semigroup $\mathcal{L}$. The zero in $\mathcal{L}$ is the filter $S$, so

$$
\operatorname{Prim}(\mathcal{L})=\{F \in \mathcal{L}: F \text { is primitive }\} \cup\{S\}
$$

Thus, to show $\operatorname{Prim}(\mathcal{L})=\mathcal{U} \cup\{S\}$, it suffices to show a filter is primitive if, and only if, the filter is an ultrafilter. Notice the similarilty between Equations (2.1), defining ultrafilters, and (3.1), defining primitive elements, so the equivalence is immediate from Lemma 2.2.9, which tells us that the natural partial order $\leq_{\mathcal{L}}$ on $\mathcal{L}$ is reverse inclusion. Then, it follows from Law98, Proposition 9.2.1, pp. 278] that $\operatorname{Prim}(\mathcal{L})$ is an inverse semigroup.

The inverse semigroup $\operatorname{Prim}(\mathcal{L})$ has an associated groupoid $\operatorname{Prim}(\mathcal{L})$.

Lemma 3.1.4. The subset $\mathcal{U}$ is a subgroupoid of the groupoid $\operatorname{Prim}(\mathcal{L})$.

Proof. Fix $U, V \in \mathcal{U}$. Suppose $U^{*} \subseteq F$ for some $F \in \mathcal{F}$. Then, $U=\left(U^{*}\right)^{*} \subseteq$ $F^{*}$, but $U$ is an ultrafilter, so $U=F^{*}$. Thus, $U^{*}=\left(F^{*}\right)^{*}=F$, which means $U^{*}$ is an ultrafilter.

Now suppose $(U, V) \in \operatorname{Prim}(\mathcal{L})^{(2)}$. Either $U \cdot V$ is in $\mathcal{U}$ or $U \cdot V=S$ by Lemma 3.1.3. so we need only show $U \cdot V \neq S$. (That is, Law98, Propsition 9.2.1, pp. 278] did the hard yards by eliminating the possibility that $U \cdot V$ is proper but not an ultrafilter.) Suppose for a contradiction that $U \cdot V=S$, so $0 \in U \cdot V$. Take $a \in U$. Then,

$$
0=a^{*} 0 \in U^{*} \cdot U \cdot V=V
$$

because $U$ and $V$ are composable. However, $0 \in V$ implies $V=S$, contradicting the fact that $V$ is proper. Therefore, $U \cdot V$ is in $\mathcal{U}$.

We call the subgroupoid $\mathcal{U}$ the groupoid of ultrafilters. Notice $\mathcal{U}$ is contained in $\mathcal{F}$ by definition of ultrafilters. In fact, $\mathcal{U}$ is an ideal in $\mathcal{F}$ in the following sense.

Proposition 3.1.5. For all $(F, G) \in \mathcal{F}^{(2)}$, if either $F$ or $G$ is in $\mathcal{U}$, then $F \cdot G \in \mathcal{U}$. 
Proof. We follow the argument used in Bic19, Proposition 2.39]. Take $(F, G) \in \mathcal{F}^{(2)}$. Suppose $G \in \mathcal{U}$. We show $F \cdot G \in \mathcal{U}$. Suppose for a contradiction there is $H \in \mathcal{F}$ such that $F \cdot G \subsetneq H$. Notice that

$$
\mathbf{r}(F)=\mathbf{r}(F \cdot G) \subseteq \mathbf{r}(H)
$$

because $F$ and $G$ are composable and $F \cdot G \subseteq H$, so

$$
\mathbf{r}(F) \cdot H \subseteq \mathbf{r}(H) \cdot H=H .
$$

We show $F^{*} \cdot H$ is a proper filter properly containing $G$. (It is tempting to think $F^{*} \cdot H$ is immediately proper because both $F^{*}$ and $H$ are in the groupoid $\mathcal{F}$ of proper filters, but we may not have that $\left(F^{*}, H\right) \in \mathcal{F}^{(2)}$.) Suppose for a contradiction that $F^{*} \cdot H$ is not proper. Filters are nonempty, so take $y \in F$. Then, $\mathbf{r}(F) \cdot H \subseteq H$ from above implies

$$
0=y 0 \in F \cdot F^{*} \cdot H=\mathbf{r}(F) \cdot H \subseteq H,
$$

contradicting the fact that $H$ is proper. Thus, $F^{*} \cdot H$ is proper. Observe

$$
G \subseteq\left(y^{*} y G\right)^{\uparrow} \subseteq F^{*} \cdot F \cdot G \subseteq F^{*} \cdot H
$$

so the proper filter $F^{*} \cdot H$ contains $G$. Since $F \cdot G \subsetneq H$, there is $x \in H \backslash F \cdot G$. Notice $y^{*} x \in F^{*} \cdot H$. Suppose for a contradiction that $y^{*} x \in G$. One of our rudiments from Lemma 2.1 .14 gives us

$$
F \cdot G \ni y y^{*} x \leq x
$$

which implies $x \in F \cdot G$, contradicting our choice of $x$. Hence, the proper filter $F^{*} \cdot H$ properly contains $G$, but then $G$ could not be an ultrafilter. Therefore, no proper filter properly contains $F \cdot G$, making $F \cdot G$ an ultrafilter.

The other case follows from a similar argument.

Notice that $\mathcal{U}$ being an ideal in $\mathcal{F}$ implies $\mathcal{U}$ is a subgroupoid, so there is an alternate construction of the groupoid $\mathcal{U}$ of ultrafilters. We keep the construction of the groupoid $\mathcal{U}$ via $\operatorname{Prim}(\mathcal{L})$ as a reminder of the rich theory of inverse semigroups and the value of viewing $\mathcal{L}$ as an inverse semigroup. 


\subsubsection{Groupoids of germs}

Groupoids of germs are well documented [Exe08]. We give an abridged construction of a particular groupoid of germs that is the range of the topological isomorphism $\pi: \mathcal{F} \rightarrow \mathcal{G}_{0}$.

The initial data for a groupoid of germs comes from an action of an inverse semigroup on a topological space. In Section 3.2.1, we will treat the set $\mathrm{F}(E)$ of proper filters in $E$ as a topological space. We are exclusively interested in the standard action of $S$ on $\mathrm{F}(E)$ from [EP16, §3]. We discuss the standard action without defining actions of inverse semigroups in general. For an extensive treatment of groupoids of germs, we refer to Exe08.

For all $e \in E$, we write $\mathrm{F}_{e}:=\{\xi \in \mathrm{F}(E): e \in \xi\}$. Define

$$
\Omega:=\left\{(s, \xi) \in S \times \mathrm{F}(E): \xi \in \mathrm{F}_{s^{*}}\right\}
$$

and define a relation $\sim$ on $\Omega$ by $(s, \xi) \sim(t, \eta)$ if, and only if, there is $e \in \xi=\eta$ such that $s e=t e$. The relation $\sim$ is an equivalence relation on $\Omega$ [EP16, §3]. We denote the equivalence class of $(s, \xi)$ by $[s, \xi]$, and we denote the set of equivalence classes by $\mathcal{G}_{0}$. For each $s \in S$ and $\xi \in \mathrm{F}_{s^{*} s}$, define

$$
\beta_{s}(\xi):=\left\{f \in E: \exists e \in \xi\left(\text { ses }^{*} \leq f\right)\right\}
$$

as per EP16, Equation (3.4)]. Define

$$
\mathcal{G}_{0}^{(2)}:=\left\{([s, \xi],[t, \eta]) \in \mathcal{G}_{0} \times \mathcal{G}_{0}: \xi=\beta_{t}(\eta)\right\} .
$$

The set $\mathcal{G}_{0}$ with the subset $\mathcal{G}_{0}^{(2)} \subseteq \mathcal{G}_{0} \times \mathcal{G}_{0}$ and the operations

$$
\left(\left[s, \beta_{t}(\eta)\right],[t, \eta]\right) \mapsto\left[s, \beta_{t}(\eta)\right][t, \eta]:=[s t, \eta] \text { and }[s, \xi] \mapsto[s, \xi]^{-1}:=\left[s^{*}, \beta_{s}(\xi)\right]
$$

forms a groupoid [EP16, §3, pp. 284], called the groupoid $\mathcal{G}_{0}$ of proper germs.

In the remainder of the section, we investigate how filters in $S$ are related to the structure underlying $\mathcal{G}_{0}$. We start by generalising parts of Law12, Lemma 2.18] (which has additional assumptions on $S$ ). 
Proposition 3.1.6. The map $\epsilon: \mathcal{F}^{(0)} \rightarrow \mathrm{F}(E), F \mapsto \epsilon(F):=E \cap F$, is a bijection with inverse $\epsilon^{-1}: \mathrm{F}(E) \rightarrow \mathcal{F}^{(0)}, \epsilon^{-1}(\xi)=\xi^{\uparrow}$.

Proof. We check $\epsilon$ and $\epsilon^{-1}$ are well defined. Fix $F \in \mathcal{F}^{(0)}$. As $F$ is an idempotent filter, $\epsilon(F)$ is nonempty. The subset $\epsilon(F)$ is an up-set in $E$ because $F$ is an up-set in $S$, and $\epsilon(F)$ is down-directed because $E^{\downarrow}=E$ and filters are down-directed. Hence, $\epsilon(F)$ is a filter in $E$. To see $\epsilon(F)$ is proper, suppose for a contradiction $\epsilon(F)=E$, so $0 \in \epsilon(F)=E \cap F$, but then $0 \in F$, contradicting $F$ being proper. Therefore, $\epsilon(F)$ is a proper filter in $E$. That is, $\epsilon(F) \in \mathrm{F}(E)$, and so $\epsilon: \mathcal{F}^{(0)} \rightarrow \mathrm{F}(E)$ is well defined.

Now fix $\xi \in \mathrm{F}(E)$. As $\xi$ is a nonempty subset of $\epsilon^{-1}(\xi)$, we know $\epsilon^{-1}(\xi)$ is nonempty. The subset $\epsilon^{-1}(\xi)$ is an up-set in $S$ because of the reflexivity of the natural partial order. The transitivity of the natural partial order with the down-directedness of $\xi$ implies $\epsilon^{-1}(\xi)$ is down-directed. Hence, $\epsilon^{-1}(\xi)$ is a filter in $S$. To see $\epsilon^{-1}(\xi)$ is proper, suppose for a contradiction that $\epsilon^{-1}(\xi)=S$, so $0 \in \epsilon^{-1}(\xi)$. Find $x \in \xi$ so that $x \leq 0$. Then, $x=x x^{*} 0=0$, in which case $0 \in \xi$, contradicting the fact that $\xi$ is proper. Therefore, $\epsilon^{-1}(\xi)$ is proper. Moreover, there is an idempotent in $\epsilon^{-1}(\xi)$, so $\epsilon^{-1}(\xi)$ is an idempotent filter. Hence, $\epsilon^{-1}(\xi) \in \mathcal{F}^{(0)}$. Thus, $\epsilon^{-1}: \mathrm{F}(E) \rightarrow \mathcal{F}^{(0)}$ is well defined.

To verify bijectivity it suffices to show $F=\epsilon(F)^{\uparrow}$ and $\xi=\epsilon\left(\xi^{\uparrow}\right)$, for all $F \in \mathcal{F}^{(0)}$ and $\xi \in \mathrm{F}(E)$. Fix $F \in \mathcal{F}^{(0)}$. Let $x \in F$. Since $F \in \mathcal{F}^{(0)}$, there is $e \in \epsilon(F)$. Because $F$ is down-directed, there is $f \in F$ such that $f \leq e, x$. As $E^{\downarrow}=E$, we know $f \in E$, so $f \in \epsilon(F)$, in which case $x \in \epsilon(F)^{\uparrow}$. Now let $x \in \epsilon(F)^{\uparrow}$, and find $e \in \epsilon(F) \subseteq F$ so that $e \leq x$. It follows that $x \in F$. Therefore, $F=\epsilon(F)^{\uparrow}$. Now fix $\xi \in \mathrm{F}(E)$. The inclusion $\xi \subseteq \epsilon\left(\xi^{\uparrow}\right)$ follows from the refexivity of the natural partial order and the fact that $\xi \subseteq E$. Also, $\xi \supseteq \epsilon\left(\xi^{\uparrow}\right)$ follows from $\xi$ being an up-set in $E$. Thus, $\xi=\epsilon\left(\xi^{\uparrow}\right)$. Therefore, $\epsilon: \mathcal{F}^{(0)} \rightarrow \mathrm{F}(E)$ is a bijection.

Define $F \mapsto \xi_{F}:=\epsilon(\mathbf{d}(F))$ from $\mathcal{F}$ to $\mathrm{F}(E)$; this is well defined by Proposition 3.1.6 since $\mathbf{d}(\mathcal{F})=\mathscr{F}^{(0)}$. 
Lemma 3.1.7. For all $F \in \mathcal{F}$ and for all $s, t \in F,\left[s, \xi_{F}\right]=\left[t, \xi_{F}\right]$.

Proof. Filters are down-directed, so there is $u \in F$ such that $u \leq s, t$. Using a characterisation of $\leq$, we have $t u^{*} u=u=s u^{*} u$, so $t u^{*} u=s u^{*} u$. Also, $u^{*} u \in \mathbf{d}(F) \cap E=\epsilon(\mathbf{d}(F))=\xi_{F}$. Therefore, $\left[s, \xi_{F}\right]=\left[t, \xi_{F}\right]$.

Lemma 3.1.8. For all $F \in \mathcal{F}$ and for all $s \in F, \beta_{s}\left(\xi_{F}\right)=\xi_{F^{*}}$.

Proof. Fix $F \in \mathcal{F}$ and let $s \in F$. We show $\xi_{F^{*}} \subseteq \beta_{s}\left(\xi_{F}\right)$. First we claim $\mathbf{r}(F) \subseteq \epsilon^{-1}\left(\beta_{s}\left(\xi_{F}\right)\right)$. To prove the claim, fix $x \in \mathbf{r}(F)$. Filters are downdirected, so there is $a \in F$ such that $a a^{*} \leq x$, and there is $b \in F$ such that $b \leq a, s$. Compatibility of the natural partial order with multiplication and inversion implies

$$
\mathbf{d}(F) \ni b^{*} b=b^{*} b b^{*} b \leq s^{*} a a^{*} s,
$$

which implies $s^{*} a a^{*} s \in \mathbf{d}(F)$. Lemma 2.1.14](3) implies $s^{*} a a^{*} s \in E$, so $s^{*} a a^{*} s \in \epsilon(\mathbf{d}(F))=\xi_{F}$. Notice

$$
s\left(s^{*} a a^{*} s\right) s^{*}=s s^{*}\left(a a^{*}\right)\left(s s^{*}\right)=s s^{*} s s^{*} a a^{*}=s s^{*} a a^{*} \leq a a^{*},
$$

from which it follows that $a a^{*} \in \beta_{s}\left(\xi_{F}\right)$. Since $a a^{*} \leq x$, we have $x \in$ $\beta_{s}\left(\xi_{F}\right)^{\uparrow}=\epsilon^{-1}\left(\beta_{s}\left(\xi_{F}\right)\right)$. Thus, $\mathbf{r}(F) \subseteq \epsilon^{-1}\left(\beta_{s}\left(\xi_{F}\right)\right)$, which implies $\epsilon(\mathbf{r}(F)) \subseteq$ $\beta_{s}\left(\xi_{F}\right)$ since $\epsilon$ is a bijection (Proposition 3.1.6). Then,

$$
\xi_{F^{*}}=\epsilon\left(\mathbf{d}\left(F^{*}\right)\right)=\epsilon(\mathbf{r}(F)) \subseteq \beta_{s}\left(\xi_{F}\right) .
$$

It remains to show $\xi_{F^{*}} \supseteq \beta_{s}\left(\xi_{F}\right)$. Fix $f \in \beta_{s}\left(\xi_{F}\right) \subseteq E$, so ses ${ }^{*} \leq f$ for some $e \in \xi_{F}$. Notice $s^{*} \in F^{*}$ and $e \in \xi_{F}=\epsilon(\mathbf{d}(F)) \subseteq \mathbf{d}(F)$, so

$$
f \geq \operatorname{ses}^{*} \in F \cdot \mathbf{d}(F) \cdot F^{*}=F \cdot F^{*}=\mathbf{r}(F) .
$$

Then, $f \in \mathbf{r}(F)^{\uparrow}=\mathbf{r}(F)=\mathbf{d}\left(F^{*}\right)$, and $f \in E$, so

$$
f \in E \cap \mathbf{d}\left(F^{*}\right)=\epsilon\left(\mathbf{d}\left(F^{*}\right)\right)=\xi_{F^{*}}
$$

Therefore, $\xi_{F^{*}} \supseteq \beta_{s}\left(\xi_{F}\right)$. 


\section{2 Étale groupoids of filters and germs}

We give topologies on $\mathrm{F}(E)$, and we describe topologies on the groupoids $\mathcal{F}$ and $\mathcal{G}_{0}$ such that both are étale groupoids. The tools we develop while showing $\mathcal{F}$ is étale prepare us to describe $\pi: \mathcal{F} \rightarrow \mathcal{G}_{0}$.

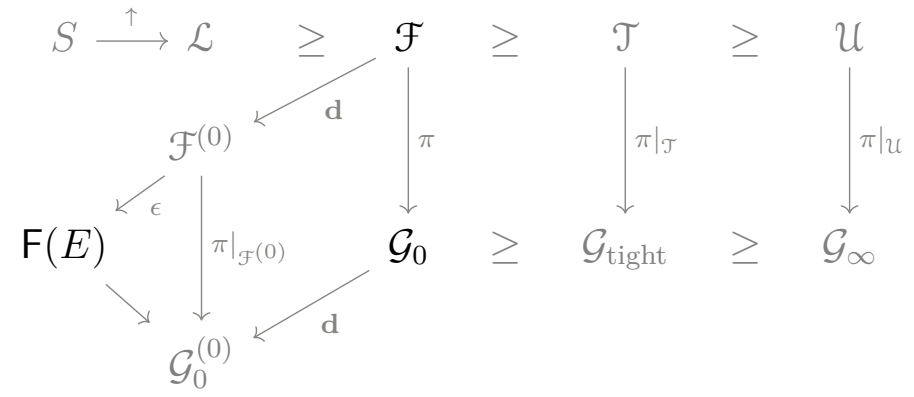

\subsubsection{Topologies on filters of idempotents}

We refer the reader to Mun00 for rudiments of point-set topology.

Let $2:=\{0,1\}$. The topologies on $\mathrm{F}(E)$ that we consider arise from topologies on $\mathbf{2}$. There are three topologies up to homeomorphism on $\mathbf{2}$ : the trivial topology $\{\emptyset, 2\}$, the Sierpiński topology $\left.\right|^{2}\{\emptyset,\{1\}, 2\}$ and the discrete topology $\{\emptyset,\{0\},\{1\}, \mathbf{2}\}$. The topologies are given in order of separability: the trivial topology is not $T_{0}$, the Sierpiński topology is $T_{0}$ but not $T_{1}$, and the discrete topology is $T_{2}$. The Sierpiński and discrete topologies are separable enough to continue investigating.

Let $\mathbf{2}^{E}$ be the power set of $E$. Notice $\mathbf{2}^{E}$ is in bijective correspondence with $\prod_{e \in E} \mathbf{2}$. Via this identification, we can endow $\mathbf{2}^{E}$ with the product topologies on $\prod_{e \in E} \mathbf{2}$.

\section{Patch topology}

Consider the product topology on $\mathbf{2}^{E}$ with respect to the discrete topology on $\mathbf{2}$, which is called the patch topology and denoted by $\tau_{\text {patch }}$. Subspaces of

\footnotetext{
${ }^{2}$ The Sierpiński topology is named after Wacław Sierpiński Sie34].
} 
$\mathbf{2}^{E}$ with respect to $\tau_{\text {patch }}$ will have the same name and notation. For example, the topology $\tau_{\text {patch }}$ on $\mathrm{F}(E)$ is the subspace topology with respect to $\tau_{\text {patch }}$ on $2^{E}$.

If $X$ is a set, we write $A \subseteq$ fin $X$ when $A$ is a finite subset of $X$. The collection of subsets of $2^{E}$ of the form

$$
\left\{\xi \in \mathbf{2}^{E}: X \subseteq \xi \subseteq E \backslash Y\right\},
$$

where $X, Y \subseteq_{\text {fin }} E$, is a basis for $\tau_{\text {patch }}$ on $\mathbf{2}^{E}$ because $\tau_{\text {patch }}$ is the product topology with respect to the discrete topology on $\mathbf{2}$. Then, the collection of subsets of $\mathrm{F}(E)$ of the form

$$
\mathrm{F}_{X: Y}:=\left\{\xi \in \mathbf{2}^{E}: X \subseteq \xi \subseteq E \backslash Y\right\} \cap \mathrm{F}(E),
$$

where $X, Y \subseteq_{\text {fin }} E$, is a basis for $\tau_{\text {patch }}$ on $\mathrm{F}(E)$. For all $X \subseteq_{\text {fin }} E$ and for all $e \in E$, define $\mathrm{F}_{e: X}:=\mathrm{F}_{\{e\}: X}$.

Lemma 3.2.1. The collection $\left(\mathrm{F}_{e: X}\right)_{e \in E, X \subseteq_{\text {fin }} \downarrow}$ is a basis for $\tau_{\text {patch }}$ on $\mathrm{F}(E)$.

Proof. The following argument elaborates on the strategy in [Law12, pp. 16]. Fix $X, Y \subseteq_{\text {fin }} E$ for an arbitrary basic open set $\mathrm{F}_{X: Y}$ in $\mathrm{F}(E)$. Let $\xi \in \mathrm{F}_{X: Y}$. Recall that, for all $e, f \in E$, ef is the meet of $e$ and $f$. Since $X$ is finite, the product $f:=\prod_{x \in X} x$ is the greatest lower bound of $X$. Then, $f \in \xi$ because $X \subseteq_{\text {fin }} \xi$ and $\xi$ is a down-directed up-set. Hence, $\xi \in \mathrm{F}_{f: Y}$. A short proof by contradiction shows that $\xi \in \mathrm{F}_{f: f Y}$ because $\xi$ is an up-set and $f Y \subseteq Y^{\downarrow}$. Also, $f Y \subseteq_{\text {fin }} f^{\downarrow}$, so we have $\mathrm{F}_{f: f Y} \in\left(\mathrm{F}_{e: X}\right)_{e \in E, X \subseteq_{\text {fin }} \downarrow}$. Since filters are downdirected up-sets and $f y$ is the meet of $f$ and $y$, for all $y \in Y$, we find that $\mathrm{F}_{f: f Y} \subseteq \mathrm{F}_{X: Y}$. Hence,

$$
\xi \in \mathrm{F}_{f: f Y} \subseteq \mathrm{F}_{X: Y} .
$$

Therefore, $\left(\mathrm{F}_{e: X}\right)_{e \in E, X \subseteq_{\text {fin }} e^{\downarrow}}$ is a basis for $\tau_{\text {patch }}$ on $\mathrm{F}(E)$.

The set $\mathbf{2}$ with discrete topology is Hausdorff, so $\mathbf{2}^{E}$ with $\tau_{\text {patch }}$ is Hausdorff because a product of Hausdorff spaces is Hausdorff Mun00, Theorem 19.4]. Further, $\mathrm{F}(E)$ and $\mathrm{U}(E)$ with $\tau_{\text {patch }}$ are Hausdorff because subspaces of Hausdorff spaces are Hausdorff [Mun00, Theorem 17.11]. 


\section{Sierpiński topology}

While we are mainly interested in the patch topology $\tau_{\text {patch }}$, we also define a useful coarser topology $\tau_{S}$. For example, in Section 3.2 .3 below we use $\tau_{S}$ to show the groupoid $\mathcal{F}$ of proper filters is étale under both $\tau_{S}$ and $\tau_{\text {patch }}$.

Recall the Sierpiński topology on $\mathbf{2}$ is $\{\emptyset,\{1\}, \mathbf{2}\}$. Consider the product topology on $\mathbf{2}^{E}$ with respect to the Sierpiński topology on $\mathbf{2}$, which we call the Sierpinski topology and denote by $\tau_{S}$. Subspaces of $\mathbf{2}^{E}$ with respect to $\tau_{S}$ will have the same name and notation. We find a basis for $\tau_{S}$ on $\mathrm{F}(E)$. For all $X \subseteq_{\text {fin }} E$ and for all $e \in E$,

$$
\mathrm{F}_{X}:=\mathrm{F}_{X: \emptyset} \text { and } \mathrm{F}_{e}:=\mathrm{F}_{\{e\}} .
$$

Observe that, for all $X \subseteq_{\text {fin }} E$,

$$
\mathrm{F}_{X}=\left\{\xi \in \mathbf{2}^{E}: X \subseteq \xi\right\} \cap \mathrm{F}(E)
$$

and the collection of subsets of the form $\left\{\xi \in \mathbf{2}^{E}: X \subseteq \xi\right\}$ is a basis for $\tau_{S}$ on $\mathbf{2}^{E}$ since $\tau_{S}$ is the product topology on $\mathbf{2}^{E}$ with respect to the Sierpiński topology on 2. It follows that $\left(\mathrm{F}_{X}\right)_{X \subseteq_{\text {fin }} E}$ is a basis for $\tau_{S}$ on $\mathrm{F}(E)$. Because filters are down-directed up-sets, we can show the subcollection $\left(\mathrm{F}_{e}\right)_{e \in E}$ of $\left(\mathrm{F}_{X}\right)_{X \subseteq_{\text {fin }} E}$ is a basis for $\tau_{S}$ on $\mathrm{F}(E)$.

The Sierpiński topology on $\mathbf{2}$ is only $T_{0}$, so Hausdorffness is rare among subspaces of $\mathbf{2}^{E}$ with $\tau_{S}$. To see how rare Hausdorffness is in $\mathrm{F}(E)$, we characterise convergence in $\mathrm{F}(E)$ and give a sufficient condition for nonHausdorffness. A sequence $\left(A_{n}\right)_{n \in \mathbb{N}}$ of subsets of $E$ pointwise eventually contains a subset $A$ of $E$ if, for all $a \in A$, there is $N_{a} \in \mathbb{N}$ such that $n \geq N_{a}$ implies $a \in A_{n}$.

Lemma 3.2.2. A sequence $\left(\xi_{n}\right)_{n \in \mathbb{N}}$ in $\mathrm{F}(E)$ converges to $\xi$ with respect to $\tau_{S}$ if, and only if, $\xi$ is pointwise eventually contained in $\left(\xi_{n}\right)_{n \in \mathbb{N}}$.

Proof. Suppose $\left(\xi_{n}\right)_{n \in \mathbb{N}}$ converges to $\xi$ with respect to $\tau_{S}$. Fix $e \in \xi$. Notice $\mathrm{F}_{e}$ is a neighbourhood of $\xi$, so there is $N_{e} \in \mathbb{N}$ such that $n \geq N_{e}$ implies 
$\xi_{n} \in \mathrm{F}_{e}$ by definition of convergence. That is, $n \geq N_{e}$ implies $e \in \xi_{n}$. Therefore, $\xi$ is pointwise eventually contained in $\left(\xi_{n}\right)_{n \in \mathbb{N}}$.

Suppose $\xi$ is pointwise eventually contained in $\left(\xi_{n}\right)_{n \in \mathbb{N}}$. Let $O$ be a neighbourhood of $\xi$. Since $\left(\mathrm{F}_{e}\right)_{e \in E}$ is a basis for $\tau_{S}$ on $\mathrm{F}(E)$, we have $O=\bigcup_{e \in A} \mathrm{~F}_{e}$ for some $A \subseteq E$, so $\xi \in \mathrm{F}_{e}$ for some $e \in A$. That is, $e \in \xi$, so there is $N_{e} \in \mathbb{N}$ such that $n \geq N_{e}$ implies $e \in \xi_{n}$. That is, $n \geq N_{e}$ implies $\xi_{n} \in \mathrm{F}_{e} \subseteq O$. Hence, $\left(\xi_{n}\right)_{n \in \mathbb{N}}$ converges to $\xi$ with respect to $\tau_{S}$.

Proposition 3.2.3. The set $\mathrm{F}(E)$ with $\tau_{S}$ is non-Hausdorff, provided there are distinct $e, f \in E$ such that ef $\neq 0$.

Proof. Suppose there are distinct $e, f \in E$ such that ef $\neq 0$. We know ef $\leq e$ and $e f \leq f$ by Lemma 2.1.14, so $e \neq f$ implies that either $e f \neq e$ or ef $\neq f$. Otherwise, antisymmetry would imply $e=f$. We assume without loss of generality that $e f \neq e$. Notice $e^{\uparrow}$ and $(e f)^{\uparrow}$ are in $\mathrm{F}(E)$ because $e$ and ef are nonzero so the filters are proper. Since $e f \leq e$, we know $e^{\uparrow} \subseteq(e f)^{\uparrow}$. Denote by $\left((e f)^{\uparrow}\right)$ the constant sequence of $(e f)^{\uparrow}$ in $\mathrm{F}(E)$. Observe the sequence $\left((e f)^{\uparrow}\right)$ pointwise eventually contains both $e^{\uparrow}$ and $(e f)^{\uparrow}$, so $\left((e f)^{\uparrow}\right)$ converges to both $e^{\uparrow}$ and $(e f)^{\uparrow}$ by Lemma 3.2.2. But $e^{\uparrow}$ and $(e f)^{\uparrow}$ are distinct because $e^{\uparrow}=(e f)^{\uparrow}$ would imply $e f=e$. Therefore, $\mathbf{F}(E)$ is non-Hausdorff since convergent sequences in Hausdorff spaces have unique limits Mun00, Theorem 17.10].

Example 3.2.4. Recall from Example 2.1.8 that there is an inverse semigroup of partial bijections associated to each set. Consider the inverse semigroup $\mathcal{J}(\mathbf{2})$ of partial bijections of $\mathbf{2}$. The empty map $0_{\mathbf{2}}$ is the zero in the inverse semigroup $\mathcal{J}(\mathbf{2})$. Denote by $E(\mathcal{J}(\mathbf{2}))$ the idempotents in $\mathcal{J}(\mathbf{2})$. Then, the identity $\operatorname{maps} \operatorname{id}_{\{0\}}$ on $\{0\}$ and $\operatorname{id}_{\mathbf{2}}$ on $\mathbf{2}$ are distinct in $E(\mathcal{J}(\mathbf{2}))$ and $\operatorname{id}_{\{0\}} \mathrm{id}_{\mathbf{2}}=$ $\operatorname{id}_{\{0\}} \neq 0_{\mathbf{2}}$. Hence, the set of filters in $E(\mathcal{J}(\mathbf{2}))$ with $\tau_{S}$ is non-Hausdorff by Proposition 3.2.3.

Though the Sierpiński topology is usually too coarse to make proper filters Hausdorff (Proposition 3.2.3), ultrafilters in $E$ are always Hausdorff. Define $\mathrm{U}_{e}:=\mathrm{F}_{e} \cap \mathrm{U}(E)$, for all $e \in E$, and define $A^{\uparrow_{E}}:=A^{\uparrow} \cap E$, for any $A \subseteq E$. 
We denote the set of filters in $E$ by $\mathrm{L}(E)$. We add details to the proof of Law10a, Lemma 2.3] in our proof of the following lemma.

Lemma 3.2.5. The set $\mathrm{U}(E)$ with $\tau_{S}$ is Hausdorff.

Proof. We show $\mathrm{U}(E)$ with $\tau_{S}$ is Hausdorff by definition. Fix distinct $\xi, \eta \in$ $\mathrm{U}(E)$. Filters are nonempty, so take $e \in \xi$ and $f \in \eta$. Observe that

$$
\xi \subseteq(\xi f)^{\uparrow_{E}} \subseteq(\xi \eta)^{\uparrow_{E}} \in \mathrm{L}(E) \text { and } \eta \subseteq(e \eta)^{\uparrow_{E}} \subseteq(\xi \eta)^{\uparrow_{E}} \in \mathrm{L}(E)
$$

Since $\xi$ and $\eta$ are ultrafilters, either $\xi=(\xi \eta)^{\uparrow_{E}}$ or $(\xi \eta)^{\uparrow_{E}}=E$, and either $\eta=(\xi \eta)^{\uparrow_{E}}$ or $(\xi \eta)^{\uparrow_{E}}=E$. Because $\xi$ and $\eta$ are distinct, it follows that $(\xi \eta)^{\uparrow_{E}}=E$. Thus, $0 \in(\xi \eta)^{\uparrow_{E}}$, so there are $x \in \xi$ and $y \in \eta$ such that $x y \leq 0$, in which case $x y=0$. If $\mu \in \mathrm{U}_{x} \cap \mathrm{U}_{y}$, then $0=x y \in \mu=E$, contradicting $\mu \in \mathrm{U}(E)$. Therefore, $\mathrm{U}_{x} \cap \mathrm{U}_{y}=\emptyset$. Since $\mathrm{U}_{x}$ and $\mathrm{U}_{y}$ are neighbourhoods of $\xi$ and $\eta$ in $\tau_{S}$, respectively, it follows that $\mathrm{U}(E)$ is Hausdorff.

Recall that $\mathrm{U}(E)$ is Hausdorff with respect to $\tau_{\text {patch }}$ too. Hence, the patch and Sierpiński topologies agree on the Hausdorff property within $\mathrm{U}(E)$. In fact, the topologies coincide on $\mathrm{U}(E)$.

Lemma 3.2.6 (Lemma 2.26 of Law12]). The topologies $\tau_{\text {patch }}$ and $\tau_{S}$ on $\mathrm{U}(E)$ are equal.

Thus, Lemma 3.2.5 is a consequence of Lemma 3.2.6.

\subsubsection{Patch topological groupoid of filters}

Recall, for any set $X$, we write $A \subseteq$ fin $X$ when $A$ is a finite subset of $X$. For all $x \in S$ and for all $T \subseteq$ fin $x^{\downarrow}$, define

$$
\mathcal{F}_{x: T}:=\{F \in \mathcal{F}: x \in F \subseteq S \backslash T\} .
$$

Recall the source map $\mathbf{d}: S \rightarrow E$ defined by $\mathbf{d}(x)=x^{*} x$. The proof of Proposition 3.2.7 mimics the argument used for [Len08, Proposition 4.1]. 
Proposition 3.2.7. The collection $\left(\mathcal{F}_{x: T}\right)_{x \in S, T \subseteq_{\text {fin }} \downarrow}$ is a basis for a topology on $\mathcal{F}$.

Proof. Fix $F \in \mathcal{F}$. Filters are nonempty, so there is $x \in F$, in which case $F \in \mathcal{F}_{x}$. Suppose $F \in \mathcal{F}_{x: A} \cap \mathcal{F}_{y: B}$. Find $z \in F$ such that $z \leq x, y$. Put $C:=z \mathbf{d}(A \cup B)$. We show $F \in \mathcal{F}_{z: C} \subseteq \mathcal{F}_{x: A} \cap \mathcal{F}_{y: B}$.

We know $z \in F$. Suppose for a contradiction there is $c \in F \cap C$. By definition of $C$, either $c=z a^{*} a$ for some $a \in A$ or $c=z b^{*} b$ for some $b \in B$. Suppose the former. Then,

$$
a=x a^{*} a \geq z a^{*} a=c \in F,
$$

so $a \in F^{\uparrow}=F$, contradicting $F \in \mathcal{F}_{x: A}$. Similarly, $c=z b^{*} b$ for some $b \in B$ yields a contradiction with $F \in \mathcal{F}_{y: B}$. Therefore, $F \cap C$ is empty, and so $F \in \mathcal{F}_{z: C}$.

We show $\mathcal{F}_{z: C} \subseteq \mathcal{F}_{x: A} \cap \mathcal{F}_{y: B}$. Fix $G \in \mathcal{F}_{z: C}$. Notice $z \leq x, y$, so $x, y \in$ $F^{\uparrow}=G$. Suppose for a contradiction there is $a \in G \cap A$. Then,

$$
z a^{*} a \in G G^{*} G=G,
$$

using Lemma 2.2.5, but $z a^{*} a \in C$ and $G \in \mathcal{F}_{z: C}$, so we have a contradiction. Thus, $G \in \mathcal{F}_{x: A}$. Similarly, $G \in \mathcal{F}_{y: B}$. Therefore, $\mathcal{F}_{z: C} \subseteq \mathcal{F}_{x: A} \cap \mathcal{F}_{y: B}$.

Recall from Lemma 3.2.1 that $\left(\mathrm{F}_{e: X}\right)_{e \in E, X \subseteq_{\text {fin }} e^{\downarrow}}$ is basis for $\tau_{\text {patch }}$ on $\mathrm{F}(E)$. Motivated by the apparent similarity of the bases, we recycle terminology and call the topology on $\mathcal{F}$ generated by $\left(\mathcal{F}_{x: T}\right)_{x \in S, T \subseteq_{\text {fin }}{ }^{\downarrow}}$ the patch topology $\tau_{\text {patch }}$.

A groupoid $\mathcal{G}$ is topological if $\mathcal{G}$ is a topological space in which composition and inversion are continuous. The source and range maps $\mathbf{d}, \mathbf{r}: \mathcal{G} \rightarrow \mathcal{G}$ are continuous too because compositions of continuous functions are continuous.

We aim to show the groupoid $\mathcal{F}$ with $\tau_{\text {patch }}$ is topological. Showing filter composition is continuous will require the following transcedence of composability of filters to the filters' members. 
Lemma 3.2.8. For all $(F, G) \in \mathcal{F}^{(2)}$ and for all $(f, g) \in F \times G$, there is $(a, b) \in F \times G$ such that

$$
a b=f g \text { and } a^{*} a=b b^{*} .
$$

Proof. Put $a:=f g g^{*}$ and $b:=f^{*} f g$ so that $a b=f g$, using the commutativity of idempotents. Notice

$$
a^{*} a=\left(f g g^{*}\right)^{*} f g g^{*}=g g^{*} f^{*} f g g^{*}=f^{*} f g g^{*} g g^{*}=f^{*} f g g^{*}
$$

and

$$
b b^{*}=f^{*} f g\left(f^{*} f g\right)^{*}=f^{*} f g g^{*} f^{*} f=f^{*} f f^{*} f g g^{*}=f^{*} f g g^{*} .
$$

Hence, $a^{*} a=b b^{*}$. It remains to show $(a, b) \in F \times G$, which is where we need the composability of $F$ and $G$. Notice

$$
a=f g g^{*} \in F \cdot \mathbf{r}(G)=F \cdot \mathbf{d}(F)=F
$$

Similarly, $b \in G$. Therefore, $(a, b) \in F \times G$.

Proposition 3.2.9. The groupoid $\mathcal{F}$ with $\tau_{\text {patch }}$ is topological.

Proof. We show filter inversion on $\mathcal{F}$ with the patch topology is continuous. Fix $x \in S$ and $T \subseteq_{\text {fin }} x^{\downarrow}$ for an arbitrary basic open set $\mathcal{F}_{x: T}$ in $\mathcal{F}$. Observe the preimage of $\mathcal{F}_{x: T}$ under inversion is $\mathcal{F}_{x^{*}: T^{*}}$, which is open.

We show filter composition from $\mathcal{F}^{(2)}$ to $\mathcal{F}$ is continuous, where $\mathcal{F}$ has the patch topology and $\mathcal{F}^{(2)}$ has the subspace topology relative to the product topology on $\mathcal{F} \times \mathcal{F}$. Our argument mimics that of [Len08, Proposition 4.3]. Recall that $\mathbf{d}(x)=x^{*} x$ and $\mathbf{r}(x)=x x^{*}$, for all $x \in S$, and denote filter composition by $\mathrm{m}: \mathcal{F}^{(2)} \rightarrow \mathcal{F}$. We show basic open sets have open preimages. Fix $x \in S$ and $T \subseteq_{\text {fin }} x^{\downarrow}$ for an arbitrary basic open set $\mathcal{F}_{x: T}$ in $\mathcal{F}$. Take any $(F, G) \in \mathrm{m}^{-1}\left(\mathcal{F}_{x: T}\right)$, so $F \cdot G \in \mathcal{F}_{x: T}$. Find $f \in F$ and $g \in G$ so that $f g \leq x$. We show $F \in \mathcal{F}_{f: \mathbf{r}(T) f}$. Suppose for a contradiction there is $t \in T$ such that $t t^{*} f \in F$. If so,

$$
t=t t^{*} x \geq t t^{*} f g \in F G
$$


because $t \leq x$, but then $t \in F \cdot G$, contradicting $F \cdot G \in \mathcal{F}_{x: T}$. Thus, $F \subseteq S \backslash \mathbf{r}(T) f$, and so $F \in \mathcal{F}_{f: \mathbf{r}(T) f}$. Similarly, $G \in \mathcal{F}_{g: g d(T)}$. Hence, $(F, G) \in$ $\mathcal{F}_{f: \mathbf{r}(T) f} \times \mathcal{F}_{g: g \mathbf{d}(T)} \cap \mathcal{F}^{(2)}$. We show $\mathcal{F}_{f: \mathbf{r}(T) f} \times \mathcal{F}_{g: g \mathbf{d}(T)} \cap \mathcal{F}^{(2)} \subseteq \mathrm{m}^{-1}\left(\mathcal{F}_{x: T)}\right.$. Fix $(H, I) \in \mathcal{F}_{f: \mathbf{r}(T) f} \times \mathcal{F}_{g: g \mathbf{d}(T)} \cap \mathcal{F}^{(2)}$. Observe $x \geq f g \in H I$, so $x \in H \cdot I$. It remains to show $H \cdot I \subseteq S \backslash T$. Suppose for a contradiction there is $t \in H \cdot I \cap T$, and find $h \in H$ and $i \in I$ so that $h i \leq t$. Since $h, f \in H$ and $i, g \in I$, there are $j \in H$ and $k \in I$ such that $j \leq h, f$ and $k \leq i, g$. By the transcendence of composability from filters to filters members (Lemma 3.2.8), we assume without loss of generality that $j^{*} j=k k^{*}$. Compute

$$
j \leq j k k^{*} f^{*} f \leq h i k^{*} f^{*} f \leq t g^{*} f^{*} f=\left(t t^{*} x\right)(f g)^{*} f \leq t t^{*} x x^{*} f \leq t t^{*} f,
$$

so $t t^{*} f \geq f \in H$ implies $t t^{*} f \in H$, contradicting $H \in \mathcal{F}_{f: \mathbf{r}(T) f}$. Thus, $H \cdot I \subseteq S \backslash T$, which means $(H, I) \in \mathrm{m}^{-1}\left(\mathcal{F}_{x: T}\right)$, and so $\mathcal{F}_{f: \mathbf{r}(T) f} \times \mathcal{F}_{g: g \mathbf{d}(T)} \cap$ $\mathcal{F}^{(2)} \subseteq \mathrm{m}^{-1}\left(\mathcal{F}_{x: T}\right)$. That is, for all $(F, G) \in \mathrm{m}^{-1}\left(\mathcal{F}_{x: T}\right)$, there are $f, g \in S$ such that

$$
(F, G) \in \mathcal{F}_{f: \mathbf{r}(T) f} \times \mathcal{F}_{g: g \mathrm{~d}(T)} \cap \mathcal{F}^{(2)} \subseteq \mathrm{m}^{-1}\left(\mathcal{F}_{x: T}\right) .
$$

It follows that $\mathrm{m}^{-1}\left(\mathcal{F}_{x: T}\right)$ is a union of basic open sets, so $\mathrm{m}^{-1}\left(\mathcal{F}_{x: T}\right)$ is open. Therefore, $\mathrm{m}$ is continuous.

The topological groupoid $\mathcal{F}$ with $\tau_{\text {patch }}$ will be the domain of $\pi: \mathcal{F} \rightarrow \mathcal{G}_{0}$.

\subsection{3 Étale groupoids}

Let $\mathcal{G}$ be a groupoid. Given subsets $A$ and $B$ of $\mathcal{G}$, we define

$$
A B:=\left\{\alpha \beta:(\alpha, \beta) \in \mathcal{G}^{(2)}\right\} .
$$

A subset $B$ of $\mathcal{G}$ is called a local bisection if $B^{-1} B$ and $B B^{-1}$ are subsets of $\mathcal{G}^{(0)}$ BS19, Definition 6.2] $]^{3}$

Lemma 3.2.10. If $B$ is a local bisection in $\mathcal{G}$, then the source map $\mathbf{d}$ is injective on $B$.

\footnotetext{
"Some authors just say "bisection" for brevity LL13.
} 
Proof. Fix $\alpha, \beta \in B$. Since $B$ is a local bisecton, $\alpha \beta^{-1}$ is in $\mathcal{G}^{(0)}$. Suppose $\mathbf{d}(\alpha)=\mathbf{d}(\beta)$. Then, $\alpha$ and $\beta^{-1}$ are composable, so

$$
\alpha=\alpha\left(\beta^{-1} \beta\right)=\left(\alpha \beta^{-1}\right) \beta=\beta
$$

because $\alpha \beta^{-1}$ is in $\mathcal{G}^{(0)}$. Hence, the restriction of $\mathbf{d}$ to $B$ is an injection.

A groupoid $\mathcal{G}$ is said to be étale if $\mathcal{G}$ is topological and the source map $\mathbf{d}: \mathcal{G} \rightarrow \mathcal{G}$ is a local homeomorphism [BS19, Definition 6.1].

Remark 3.2.11. The literature contains various definitions of étale groupoids. For disambiguation, note that it is equivalent to say that a groupoid $\mathcal{G}$ is étale if $\mathcal{G}$ is topological and, for each $\gamma \in \mathcal{G}$, there is a local bisection $B_{\gamma}$ containing $\gamma$ such that both $B_{\gamma}$ and $\mathbf{d}\left(B_{\gamma}\right)$ are open in $\mathcal{G}$.

Let $\mathcal{G}$ be both a groupoid and a topological space. A basis $\mathcal{B}$ for the topology on $\mathcal{G}$ is called étale if

(1) $O^{-1} \in \mathcal{B}$,

(2) $O N \in \mathcal{B}$, and

(3) $O^{-1} O \subseteq \mathcal{G}^{(0)}$

for all $O, N \in \mathcal{B}$ BS19, Definition 6.5]. In this work, we prove groupoids are étale via étale bases using the following result.

Proposition 3.2.12 (Proposition 6.6 of BS19]). Let $\mathcal{G}$ be both a groupoid and a topological space. The topological space $\mathcal{G}$ has an étale basis if, and only if, the groupoid $\mathcal{G}$ is étale, in which case $\mathcal{G}$ is a topological groupoid.

We aim to show $\mathcal{F}$ is an étale groupoid with respect to $\tau_{\text {patch. }}$. We first show $\mathcal{F}$ is étale with respect to the coarser topology defined below, and we conclude that $\mathcal{F}$ is étale with respect to $\tau_{\text {patch }}$ as desired.

For all $x \in S$, define

$$
\mathcal{F}_{x}:=\{F \in \mathcal{F}: x \in F\}
$$


Proposition 3.2.13. The collection $\left(\mathcal{F}_{x}\right)_{x \in S}$ is a basis for a topology on $\mathcal{F}$.

Proof. Fix $F \in \mathcal{F}$. Filters are nonempty, so there is $x \in F$, in which case $F \in \mathcal{F}_{x}$. Suppose $F \in \mathcal{F}_{x} \cap \mathcal{F}_{y}$. Then, $x, y \in F$, and filters are down-directed, so there is $z \in F$ such that $z \leq x, y$. Notice $F \in \mathcal{F}_{z}$. Also, filters are up-sets, so $\mathcal{F}_{z} \subseteq \mathcal{F}_{x} \cap \mathcal{F}_{y}$.

We call the topology on $\mathcal{F}$ generated by $\left(\mathcal{F}_{x}\right)_{x \in S}$ the Sierpinsski topology and denote it by $\tau_{S}$. Items (1), (2) and (3) in Lemma 3.2.14 below are adapted from items (2), (3) and (4) in [Law12, Lemma 2.10] (which has additional assumptions on $S$ ).

Lemma 3.2.14. For all $s, t \in S$,

(1) $\mathcal{F}_{s}^{*}=\mathcal{F}_{s^{*}}$,

(2) $\mathcal{F}_{s} \mathcal{F}_{t}=\mathcal{F}_{s t}$,

(3) $\mathcal{F}_{s}$ is a local bisection in $\mathcal{F}$,

(4) $\mathbf{d}\left(\mathcal{F}_{s}\right)=\mathcal{F}_{s^{*} s}$, and

(5) $\left(\mathcal{F}_{x}\right)_{x \in S}$ is an étale basis for the topology on the groupoid $\mathcal{F}$.

Proof. Item (1) follows from the fact that $s=\left(s^{*}\right)^{*}$ and $F=\left(F^{*}\right)^{*}$, for all $F \in \mathcal{F}$, because $S$ and $\mathcal{F}$ are inverse semigroups.

(2) We follow the argument for [Law12, Lemma 2.10(4)]. Given $(F, G) \in$ $\mathcal{F}_{s} \mathcal{F}_{t}$, we have $s t \in F G \subseteq F \cdot G \in \mathcal{F}$, so $F \cdot G \in \mathcal{F}_{s t}$. Fix $H \in \mathcal{F}_{s t}$. Put $F:=\left(s\left(t \mathbf{d}(H) t^{*}\right)^{\uparrow}\right)^{\uparrow}$ and $G:=(t \mathbf{d}(H))^{\uparrow}$. The subsets $F$ and $G$ are filters by Lemma 2.2.4. We show $F$ and $G$ contain $s$ and $t$, respectively. One of the rudiments of inverse semigroups from Lemma 2.1 .14 implies $t^{*} t \geq(s t)^{*} s t \in$ $\mathbf{d}(H)$, so $t^{*} t \in \mathbf{d}(H)^{\uparrow}=\mathbf{d}(H)$. Then, $t=t t^{*} t \in(t \mathbf{d}(H))^{\uparrow}=G$ by definition of $G$. Also,

$$
s^{*} s \geq s^{*} s t t^{*} \geq t t^{*} s^{*} s t t^{*}=t(s t)^{*} s t t^{*} \in t \mathbf{d}(H) t^{*},
$$


so $s^{*} s \in\left(t \mathbf{d}(H) t^{*}\right)^{\uparrow}$. Thus, $s=s s^{*} s \in\left(s\left(t \mathbf{d}(H) t^{*}\right)^{\uparrow}\right)^{\uparrow}=F$. That is, $s \in F$ and $t \in G$.

We show $\mathbf{d}(F) \subseteq \mathbf{r}(G)$. Fix $x \in F$. It suffices to show $x^{*} x \in \mathbf{r}(G)$. Using the down-directedness of filters, we can find $h \in H$ such that $x \geq s t h^{*} h t^{*}$. Since $h, s t \in H$, we assume without loss of generality that $h \leq s t$. Then, $h=s t h^{*} h$, so $x \geq s t h^{*} h t^{*}=h t^{*}$. Then,

$$
x^{*} x \geq\left(h t^{*}\right)^{*} h t^{*}=t h^{*} h t^{*}=t h^{*} h h^{*} h t^{*}=t h^{*} h\left(t h^{*} h\right)^{*} \in \mathbf{r}(G) .
$$

Hence, $x^{*} x \in \mathbf{r}(G)^{\uparrow}=\mathbf{r}(G)$. Similar computations yield $\mathbf{d}(F) \supseteq \mathbf{r}(G)$. Therefore, $\mathbf{d}(F)=\mathbf{r}(G)$.

Next we show $F \cdot G=H$. Fix $x \in F \cdot G$. There exists $h_{1}, h_{2} \in H$ such that $\left(s t h_{1}^{*} h_{1} t^{*}\right)\left(t h_{2}^{*} h_{2}\right) \leq x$. Since $h_{1}, h_{2}, s t \in H$, there is $h \in H$ such that $h \leq h_{1}, h_{2}$, st. Observe

$$
x \geq s t h_{1}^{*} h_{1} t^{*} t h_{2}^{*} h_{2}=s t t^{*} t h_{1}^{*} h_{1} h_{2}^{*} h_{2}=s t h_{1}^{*} h_{1} h_{2}^{*} h_{2} \geq s t h^{*} h=h \in H,
$$

so $x \in H^{\uparrow}=H$. For the reverse inclusion, if $h \in H$, we can assume $h \leq s t$. Then, a similar comptuation yields $h=\left(s t h^{*} h t^{*}\right)\left(t h^{*} h\right)$ so that $h \in F \cdot G$. Hence, $F \cdot G=H$.

It remains to show $F$ and $G$ are proper so that $H=F \cdot G \in \mathcal{F}_{s} \mathcal{F}_{t}$. If either $F$ or $G$ contained 0 , then so would $H$ because $H=F \cdot G$, but $H$ is proper, so neither $F$ nor $G$ contains 0 .

(3) We show $\mathcal{F}_{x}^{*} \mathcal{F}_{x} \cup \mathcal{F}_{x} \mathcal{F}_{x}^{*} \subseteq \mathcal{F}^{(0)}$. Observe $\mathcal{F}_{x}^{*} \mathcal{F}_{x}=\mathcal{F}_{x^{*}} \mathcal{F}_{x}=\mathcal{F}_{x^{*} x}$ by (1) and (2). Similarly, $\mathcal{F}_{x} \mathcal{F}_{x}^{*}=\mathcal{F}_{x x^{*}}$, so $\mathcal{F}_{x}^{*} \mathcal{F}_{x} \cup \mathcal{F}_{x} \mathcal{F}_{x}^{*}=\mathcal{F}_{x^{*} x} \cup \mathcal{F}_{x x^{*}}$. Since idempotent proper filters are units in the groupoid $\mathcal{F}$ (see Section 3.1.2), we have $\mathcal{F}_{x}^{*} \mathcal{F}_{x} \cup \mathcal{F}_{x} \mathcal{F}_{x}^{*}=\mathcal{F}_{x^{*} x} \cup \mathcal{F}_{x x^{*}} \subseteq \mathcal{F}^{(0)}$.

(4) Observe $\mathbf{d}\left(\mathcal{F}_{x}\right)=\left\{F^{*} \cdot F: F \in \mathcal{F}_{x}\right\} \subseteq \mathcal{F}_{x^{*} x}$. For the reverse inclusion, it is enough to show $\mathbf{d}\left(\mathcal{F}_{x}\right) \supseteq \mathcal{F}_{x^{*}} \mathcal{F}_{x}$ by $(2)$, Take $F \cdot G \in \mathcal{F}_{x^{*}} \mathcal{F}_{x}$, where $(F, G) \in \mathcal{F}_{x^{*}} \times \mathcal{F}_{x} \cap \mathcal{F}^{(2)}$. Observe that $\mathbf{d}(F)=\mathbf{d}\left(G^{*}\right)$ and $F, G^{*} \in \mathcal{F}_{x^{*}}$, which is a local bisection by (3), so $F=G^{*}$ by Lemma 3.2.10. Thus,

$$
F \cdot G=G^{*} \cdot G=\mathbf{d}(G) \in \mathbf{d}\left(\mathcal{F}_{s}\right) .
$$

(5) This is a consequence of (1), (2) and (4). 
Proposition 3.2.15. The groupoid $\mathcal{F}$ of proper filters is étale with respect to both $\tau_{S}$ and $\tau_{\text {patch }}$.

Proof. It is immediate from Lemma 3.2.14) that $\mathcal{F}$ is étale with respect to $\tau_{S}$ due to Proposition 3.2 .12 , which says a groupoid with an étale basis is étale.

In Proposition 3.2.9, we showed $\mathcal{F}$ with $\tau_{\text {patch }}$ is a topological groupoid. Since $\tau_{\text {patch }}$ is finer than $\tau_{S}$ and $\mathcal{F}$ is étale with respect to $\tau_{S}$, we have that $\mathcal{F}$ is étale with respect to $\tau_{\text {patch }}$ too-there being more open sets for the same points.

Assumption 3.2.16. The groupoid $\mathcal{F}$ is endowed with $\tau_{\text {patch }}$.

The source map in $\mathcal{F}$ interacts with a basis for $\tau_{\text {patch }}$ in the following way. (Both $\mathbf{d}: \mathcal{F} \rightarrow \mathcal{F}^{(0)}$ and $\mathbf{d}: S \rightarrow E$ are used in the following result; which map is being used is implicit from the context.)

Lemma 3.2.17. For all $s \in S$ and for all $T \subseteq_{\text {fin }} s^{\downarrow}$,

$$
\mathbf{d}\left(\mathcal{F}_{s: T}\right)=\mathcal{F}_{s^{*} s: s^{*} s \mathbf{d}(T)}
$$

Proof. Take any $F \in \mathcal{F}_{s: T}$. Notice $s^{*} s \in \mathbf{d}(F)$. We show $\mathbf{d}(F) \subseteq S \backslash s^{*} s \mathbf{d}(T)$. Suppose for a contradiction that $s^{*} s t^{*} t \in \mathbf{d}(F)$ for some $t \in T$. Since $t \leq s$, we have $t=s t^{*} t$, so $s^{*} t=s^{*} s t^{*} t \in \mathbf{d}(F)$. Then, $t \in(s \mathbf{d}(F))^{\uparrow}=F$ by Lemma 2.2.11. But $t \in F$ contradicts $F \in \mathcal{F}_{s: T}$. Thus, $\mathbf{d}(F) \subseteq S \backslash s^{*} s \mathbf{d}(T)$, and so $\mathbf{d}(F) \in \mathcal{F}_{s^{*} s: s^{*} s \mathbf{d}(T)}$.

For the reverse inclusion, let $X \in \mathcal{F}_{s^{*} s: s^{*} s \mathbf{d}(T)}$. Then, $X \in \mathcal{F}_{s^{*} s}=\mathbf{d}\left(\mathcal{F}_{s}\right)$ by Lemma 3.2.14)(4), so there is $F \in \mathcal{F}_{s}$ such that $X=\mathbf{d}(F)$. It remains to show $F \in \mathcal{F}_{s: T}$. We ensured $s \in F$. Suppose for a contradiction that $t \in F$ for some $t \in T$. Then, $s^{*} s, t^{*} t \in \mathbf{d}(F)$, but that would mean $s^{*} s t^{*} t \in \mathbf{d}(F)$ since $s^{*} s t^{*} t$ is the meet of $s^{*} s$ and $t^{*} t$ (Lemma 2.1.15), contradicting $\mathbf{d}(F) \in \mathcal{F}_{s^{*} s: s^{*} s \mathbf{d}(T)}$. Therefore, $F \in \mathcal{F}_{s: T}$. 


\section{Groupoid of proper germs}

We treat the topology on the groupoid $\mathcal{G}_{0}$ of proper germs as briefly as we did the groupoid's construction in Section 3.1.3. Consider $\mathrm{F}(E)$ with $\tau_{\text {patch. }}$. The collection of subsets

$$
\Theta_{0}(x, A):=\left\{[x, \xi] \in \mathcal{G}_{0}: \xi \in A\right\},
$$

where $x \in S$ and $A \subseteq \mathrm{F}_{x^{*} x}$ is open in $\mathrm{F}(E)$, is a basis for a topology on $\mathcal{G}_{0}$ such that $\mathcal{G}_{0}$ is étale EP16, §3].

\subsection{Hausdorff unit spaces}

We show $\mathrm{F}(E), \mathcal{F}^{(0)}$ and $\mathcal{G}_{0}^{(0)}$ are homeomorphic, and we study the identification that arises between the unit spaces $\mathcal{F}^{(0)}$ and $\mathcal{G}_{0}^{(0)}$. This identification of unit spaces will be extended to the topological isomorphism $\pi: \mathcal{F} \rightarrow \mathcal{G}_{0}$.

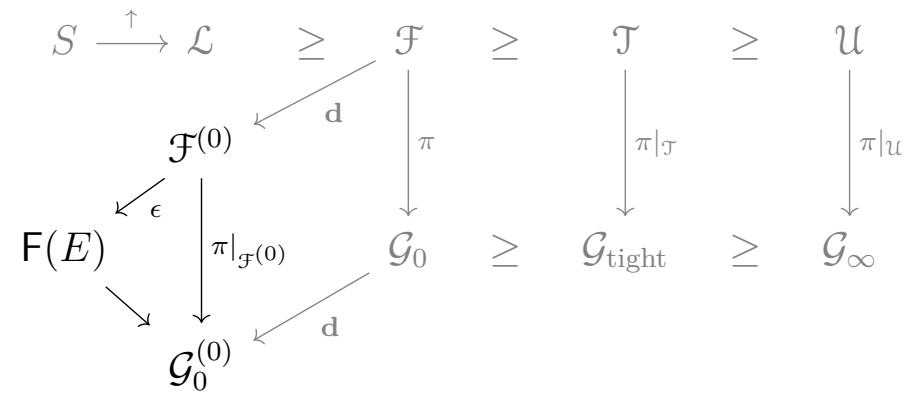

Recall from Proposition 3.1.6 that the map $\epsilon: \mathcal{F}^{(0)} \rightarrow \mathrm{F}(E)$, such that

$$
F \mapsto \epsilon(F)=E \cap F,
$$

is a bijection with inverse $\epsilon^{-1}: \mathrm{F}(E) \rightarrow \mathcal{F}^{(0)}$, such that

$$
\xi \mapsto \epsilon^{-1}(\xi)=\xi^{\uparrow} .
$$

We first aim to show $\epsilon$ is a homeomorphism. Recall from Proposition 3.2.7 that $\left(\mathcal{F}_{x: T}\right)_{x \in S, T \subseteq_{\text {fin }} \downarrow}$ is a basis for a topology on $\mathcal{F}$. Then, the collection $\left(\mathcal{F}^{(0)} \cap \mathcal{F}_{x: T}\right)_{x \in S, T \subseteq_{\text {fin }} x^{\downarrow}}$ is a basis for $\tau_{\text {patch }}$ on $\mathcal{F}^{(0)}$, but it will be helpful to have a basis indexed by idempotents. 
Lemma 3.3.1. The collection $\left(\mathcal{F}_{e: X}\right)_{e \in E, X \subseteq \text { fin }} e^{\downarrow}$ is a basis for $\tau_{\text {patch }}$ on $\mathcal{F}^{(0)}$.

Proof. We show that, for any open set $A$ in $\mathcal{F}^{(0)}$ and any filter $F$ in $A$, there is an element $B$ of $\left(\mathcal{F}_{e: X}\right)_{e \in E, X \subseteq_{\text {fin }}{ }^{\downarrow}}$ such that $F \in B \subseteq A$. Then, it will follow from Mun00, Lemma 13.2] that $\left(\mathcal{F}_{e: X}\right)_{e \in E, X \subseteq_{\text {fin }} \downarrow}$ is a basis for the topology on $\mathcal{F}^{(0)}$. Since $\left(\mathcal{F}^{(0)} \cap \mathcal{F}_{s: T}\right)_{s \in S, T \subseteq_{\text {fin }} \downarrow}$ is a basis, and open sets are unions of basis elements, it suffices to check each of the basis elements.

Fix $s \in S$ and let $T \subseteq_{\text {fin }} s^{\downarrow}$, and consider $\mathcal{F}^{(0)} \cap \mathcal{F}_{s: T}$. Let $F \in \mathcal{F}^{(0)} \cap \mathcal{F}_{s: T}$. We find $e \in E$ and $X \subseteq_{\text {fin }} e^{\downarrow}$ so that $F \in \mathcal{F}_{e: X} \subseteq \mathcal{F}^{(0)} \cap \mathcal{F}_{s: T}$. Since $F \in \mathcal{F}^{(0)}$, $F$ is an idempotent filter, so there is $e \in E \cap F$. We assume without loss of generality that $e \leq s$ because filters are down-directed and $E^{\downarrow}=E$. Put $X:=e \mathbf{d}(T)$. Notice $X \subseteq_{\text {fin }} e^{\downarrow}$ by a rudiment from Lemma 2.1.14.

We show $F \in \mathcal{F}_{e: X}$. Since $e \in F$, it remains to show $F \subseteq S \backslash X$. Suppose for a contradiction that there is $t \in T$ such that $e t^{*} t \in F$. We know $e=e s$ because $e \leq s$ and idempotents are self-inverse. Also, $t \in T \subseteq$ fin $s^{\downarrow}$ means $t \leq s$, so $s t^{*} t=t$. Hence,

$$
t \geq e t=e\left(s t^{*} t\right)=(e s) t^{*} t=e t^{*} t \in F,
$$

so $t \in F^{\uparrow}=F$, contradicting $F \subseteq S \backslash T$. Therefore, $F \in \mathcal{F}_{e: X}$.

We show $\mathcal{F}_{e: X} \subseteq \mathcal{F}^{(0)} \cap \mathcal{F}_{s: T}$. Fix $G \in \mathcal{F}_{e: X}$. Since $s \geq e \in G$, we know $s \in G$, and $G \in \mathcal{F}^{(0)}$ because $e$ is an idempotent. It remains to show $G \subseteq S \backslash T$. Suppose for a contradiction there is $t \in G \cap T$, so

$$
e t^{*} t \in G \cdot \mathbf{d}(G)=G
$$

However, $e t^{*} t \in X$ and $G \subseteq S \backslash X$. Hence, $G \subseteq S \backslash T$, as required. Therefore, $F \in \mathcal{F}_{e: X} \subseteq \mathcal{F}^{(0)} \cap \mathcal{F}_{s: T}$.

Proposition 3.3.2. The map $\epsilon: \mathcal{F}^{(0)} \rightarrow \mathrm{F}(E)$ is a homeomorphism with respect to $\tau_{\text {patch }}$, and $\left.\epsilon\right|_{\mathcal{U}^{(0)}}$ is a homeomorphism from $\mathcal{U}^{(0)}$ to $\mathrm{U}(E)$.

Proof. Observe that $\epsilon\left(\mathcal{F}_{e: X}\right)=\mathrm{F}_{e: X}$, for all $e \in E$ and for all $X \subseteq_{\text {fin }} e^{\downarrow}$. It follows that $\epsilon: \mathcal{F}^{(0)} \rightarrow \mathrm{F}(E)$ is a homeomorphism because $\left(\mathcal{F}_{e: X}\right)_{e \in E, X \subseteq_{\text {fin }}{ }^{\downarrow}}$ is 
a basis for $\tau_{\text {patch }}$ on $\mathcal{F}^{(0)}$ by Lemma 3.3.1 and $\left(\mathrm{F}_{e: X}\right)_{e \in E, X \subseteq_{\text {fin }} e^{\downarrow}}$ is a basis for $\tau_{\text {patch }}$ on $\mathrm{F}(E)$ by Lemma 3.2.1.

We show $\left.\epsilon\right|_{\mathcal{U}^{(0)}}$ is a bijection from $\mathcal{U}^{(0)}$ to $\mathrm{U}(E)$. It suffices to show $\epsilon\left(\mathcal{U}^{(0)}\right)=\mathrm{U}(E)$ because $\epsilon$ is a bijection from $\mathcal{F}^{(0)}$ to $\mathrm{F}(E)$. Fix $U \in \mathcal{U}^{(0)}$. Suppose $\epsilon(U) \subseteq \xi \in \mathrm{F}(E)$. Then, $U \subseteq \epsilon^{-1}(\xi)$ but $U$ is an ultrafilter, so $U=\epsilon^{-1}(\xi)$. Thus, $\epsilon(U)=\xi$, and so $\epsilon(U) \in \mathrm{U}(E)$. A similar argument yields the reverse inclusion, so $\epsilon\left(\mathcal{U}^{(0)}\right)=\mathrm{U}(E)$.

Furthermore, the unit space $\mathcal{G}_{0}^{(0)}$ is homeomorphic to $\mathrm{F}(E)$ via the map

$$
\xi \mapsto[e, \xi]
$$

where $e \in \xi$, from $\mathrm{F}(E)$ to $\mathcal{G}_{0}^{(0)}$ EP16, Equation (3.9)].

Proposition 3.3.3. The unit spaces $\mathcal{F}^{(0)}$ and $\mathcal{G}_{0}^{(0)}$ are Hausdorff.

Proof. Recall from Section 3.2.1 that $\mathrm{F}(E)$ with $\tau_{\text {patch }}$ is Hausdorff, and we have seen in the present section that both $\mathcal{F}^{(0)}$ and $\mathcal{G}_{0}^{(0)}$ are homeomorphic to $\mathrm{F}(E)$.

The corollary below can be shown to hold by composing the above homeomorphisms from $\mathcal{F}^{(0)}$ to $\mathrm{F}(E)$ and from $\mathrm{F}(E)$ to $\mathcal{G}_{0}^{(0)}$.

Corollary 3.3.4. The map

$$
F \mapsto[e, \epsilon(F)]
$$

where $e$ is any element of $\epsilon(F)$, from $\mathcal{F}^{(0)}$ to $\mathcal{G}_{0}^{(0)}$ is a homeomorphism.

\subsection{Filters isomorphic to germs}

We extend the homeomorphism $F \mapsto[e, \epsilon(F)]$ from $\mathcal{F}^{(0)}$ to $\mathcal{G}_{0}^{(0)}$ in Corollary 3.3.4 to the topological isomorphism $\pi: \mathcal{F} \rightarrow \mathcal{G}_{0}$. 


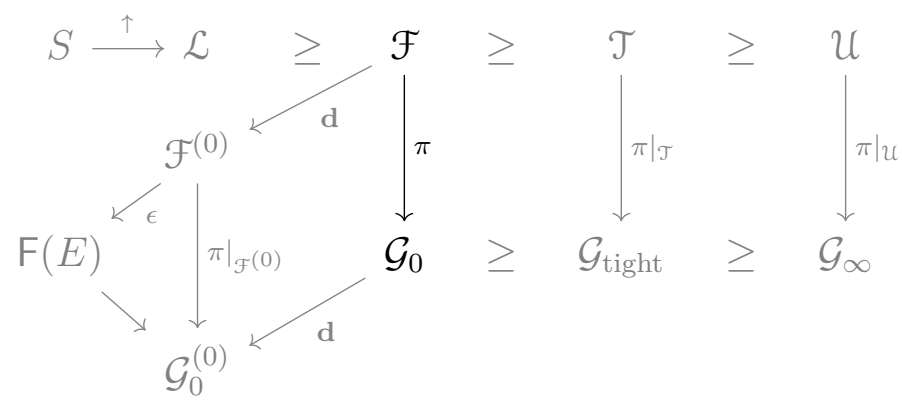

Given groupoids $\mathcal{G}$ and $\mathcal{H}$, a bijection $\phi: \mathcal{G} \rightarrow \mathcal{H}$ is called an isomorphism if $(g, h) \in \mathcal{G}^{(2)}$ implies $(\phi(g), \phi(h)) \in \mathcal{H}^{(2)}$ and $\phi(g h)=\phi(g) \phi(h)$ Sim18, $\S 2$, pp. 8]. If $\mathcal{G}$ and $\mathcal{H}$ are topological and $\phi$ is both a homeomorphism and an isomorphism, we say $\phi$ is a topological isomorphism.

Before stating the main result of this work, we recall key ingredients. For all $F \in \mathcal{F}$, we have $\xi_{F}=\epsilon(\mathbf{d}(F))=E \cap \mathbf{d}(F) \in \mathbf{F}(E)$ due to the homeomorphism $\epsilon: \mathcal{F}^{(0)} \rightarrow \mathrm{F}(E)$ from Proposition 3.3.2. In Lemmas 3.1.7 and 3.1.8, we found that:

- for all $F \in \mathcal{F}$ and for all $s, t \in F,\left[s, \xi_{F}\right]=\left[t, \xi_{F}\right]$; and

- for all $F \in \mathcal{F}$ and for all $s \in F, \beta_{s}\left(\xi_{F}\right)=\xi_{F^{*}}$.

We noted in (3) and (4) of Lemma 3.2.14 that $\mathcal{F}_{x}$ is a local bisection and $\mathcal{F}_{x^{*} x}=\mathbf{d}\left(\mathcal{F}_{x}\right)$, for all $x \in S$.

Theorem 3.4.1. The map $\pi: \mathcal{F} \rightarrow \mathcal{G}_{0}$ given by,

$$
F \mapsto \pi(F):=\left[s, \xi_{F}\right]
$$

where $s \in F$, is a topological isomorphism, and the inverse $\pi^{-1}: \mathcal{G}_{0} \rightarrow \mathcal{F}$ is given by $\pi^{-1}([s, \xi])=(s \xi)^{\uparrow}$.

Proof. Since $\left[s, \xi_{F}\right]=\left[t, \xi_{F}\right]$ for any $s, t \in F$, we know $\pi$ is well defined. We show $\pi$ is injective. Fix $F, G \in \mathcal{F}$ and take $f \in F$ and $g \in G$. Then, $\pi(F)=\left[s, \xi_{F}\right]$ and $\pi(G)=\left[t, \xi_{G}\right]$. Suppose $\left[s, \xi_{F}\right]=\left[t, \xi_{G}\right]$. We show $F=G$. By definition of the equivalence relation $\sim$ from Section 3.1.3. $\left[s, \xi_{F}\right]=\left[t, \xi_{G}\right]$ 
implies there is $e \in \xi_{F}=\xi_{G}$ such that $s e=t e$. Observe $\xi_{F}=\xi_{G}$ means $\epsilon(\mathbf{d}(F))=\epsilon(\mathbf{d}(G))$, so $\mathbf{d}(F)=\mathbf{d}(G)$ because $\epsilon$ is a bijection from $\mathcal{F}^{(0)}$ to $\mathrm{F}(E)$ by Proposition 3.3.2, Also,

$$
F=F \cdot \mathbf{d}(F) \ni s e=t e \in G \cdot \mathbf{d}(G)=G,
$$

which means $F, G \in \mathcal{F}_{\text {se }}$ and $\mathbf{d}(F)=\mathbf{d}(G)$. Notice $\mathcal{F}_{\text {se }}$ is a local bisection by Lemma 3.2.14(3), Lemma 3.2.10 says $\mathbf{d}$ is injective on $\mathcal{F}_{s e}$, so we have $F=G$. Next we show $\pi$ is surjective. Fix $[s, \xi] \in \mathcal{G}_{0}$. We know $\xi \in \mathrm{F}_{s^{*} s}$, so we have

$$
\epsilon^{-1}(\xi) \in \mathcal{F}_{s^{*} s}=\mathbf{d}\left(\mathcal{F}_{s}\right)
$$

by Lemma 3.2.14(4), As $\epsilon^{-1}(\xi) \in \mathbf{d}\left(\mathcal{F}_{s}\right)$, there is $F \in \mathcal{F}_{s}$ such that $\mathbf{d}(F)=$ $\epsilon^{-1}(\xi)$. Then, $s \in F$ and

$$
\xi_{F}=\epsilon(\mathbf{d}(F))=\epsilon\left(\epsilon^{-1}(\xi)\right)=\xi
$$

It follows that $\pi(F)=\left[s, \xi_{F}\right]=[s, \xi]$. Therefore, $\pi$ is a bijection. Further, the reconstruction of filters from sources and members (Lemma 2.2.11) implies $F=(s \mathbf{d}(F))^{\uparrow}$, so

$$
\pi^{-1}([s, \xi])=\pi^{-1}(\pi(F))=F=(s \mathbf{d}(F))^{\uparrow}=\left(s \epsilon^{-1}(\xi)\right)^{\uparrow}=\left(s \xi^{\uparrow}\right)^{\uparrow}=(s \xi)^{\uparrow} .
$$

Now we show $\pi$ is an isomorphism. Fix $(F, G) \in \mathcal{F}^{(2)}$.

We show $(\pi(F), \pi(G)) \in \mathcal{G}_{0}^{(2)}$. Say $s \in F$ and $t \in G$, so $\pi(F)=\left[s, \xi_{F}\right]$ and $\pi(G)=\left[t, \xi_{G}\right]$. Since $\mathbf{d}(F)=\mathbf{r}(G)=\mathbf{d}\left(F^{*}\right)$,

$$
\xi_{F}=\epsilon(\mathbf{d}(F))=\epsilon\left(\mathbf{d}\left(G^{*}\right)\right)=\xi_{G^{*}}=\beta_{t}\left(\xi_{G}\right)
$$

by Lemma 3.1 .8 , so $\xi_{F}=\beta_{t}\left(\xi_{G}\right)$. It follows that $(\pi(F), \pi(G)) \in \mathcal{G}_{0}^{(2)}$ by definition of $\mathcal{G}_{0}^{(2)}$.

We show $\pi(F \cdot G)=\pi(F) \pi(G)$. Notice $s t \in F \cdot G$ and

$$
\xi_{F \cdot G}=\epsilon(\mathbf{d}(F \cdot G))=\epsilon(\mathbf{d}(G))=\xi_{G},
$$

So

$$
\pi(F \cdot G)=\left[s t, \xi_{F \cdot G}\right]=\left[s t, \xi_{G}\right]=\left[s, \xi_{F}\right]\left[t, \xi_{G}\right]=\pi(F) \pi(G)
$$


Therefore, $\pi$ is an isomorphism.

We show $\pi$ is continuous by showing basic open sets in $\mathcal{G}_{0}$ have open preimages in $\mathcal{F}$. Fix $s \in S$ and take any open $A \subseteq \mathrm{F}_{s^{*} s}$ with respect to $\tau_{\text {patch }}$ for an arbitrary basic open set $\Theta_{0}(s, A)$ in $\mathcal{G}_{0}$. We show $\pi^{-1}\left(\Theta_{0}(s, A)\right)=$ $\mathcal{F}_{s} \cap \mathbf{d}^{-1}\left(\epsilon^{-1}(A)\right)$. The earlier expression for $\pi^{-1}$ gives us $\pi^{-1}\left(\Theta_{0}(s, A)\right)=$ $\left\{(s \xi)^{\uparrow}: \xi \in A\right\}$. For any $\xi \in A$, notice $s^{*} s \in \xi$. It follows that $\mathbf{d}\left((s \xi)^{\uparrow}\right)=\xi^{\uparrow}$, which implies $\epsilon\left(\mathbf{d}\left((s \xi)^{\uparrow}\right)\right)=\epsilon\left(\xi^{\uparrow}\right)=\xi \in A$. That is, $(s \xi)^{\uparrow} \in \mathbf{d}^{-1}\left(\epsilon^{-1}(A)\right)$, for all $\xi \in A$. Moreover, $s=s s^{*} s \in(s \xi)^{\uparrow}$, so $(s \xi)^{\uparrow} \in \mathcal{F}_{s}$, for all $\xi \in A$. Hence, $\left\{(s \xi)^{\uparrow}: \xi \in A\right\} \subseteq \mathcal{F}_{s} \cap \mathbf{d}^{-1}\left(\epsilon^{-1}(A)\right)$. Now fix $F \in \mathcal{F}_{s} \cap \mathbf{d}^{-1}\left(\epsilon^{-1}(A)\right)$. Then, $s \in F$ and $\xi_{F}=\epsilon(\mathbf{d}(F)) \in A$, so $\pi(F)=\left[s, \xi_{F}\right] \in \Theta_{0}(s, A)$. Thus, $F \in \pi^{-1}\left(\Theta_{0}(s, A)\right)$. Therefore,

$$
\mathcal{F}_{s} \cap \mathbf{d}^{-1}\left(\epsilon^{-1}(A)\right) \subseteq \pi^{-1}\left(\Theta_{0}(s, A)\right)=\left\{(s \xi)^{\uparrow}: \xi \in A\right\} \subseteq \mathcal{F}_{s} \cap \mathbf{d}^{-1}\left(\epsilon^{-1}(A)\right)
$$

so $\pi^{-1}\left(\Theta_{0}(s, A)\right)=\mathcal{F}_{s} \cap \mathbf{d}^{-1}\left(\epsilon^{-1}(A)\right)$. Now Proposition 3.3.2 says $\epsilon: \mathcal{F}^{(0)} \rightarrow$ $\mathrm{F}(E)$ is continuous, so $\epsilon^{-1}(A)$ is open in $\mathcal{F}^{(0)}$ because $A$ is open in $\mathrm{F}(E)$. As $\mathcal{F}$ is étale (Proposition 3.2.15), we know $\mathcal{F}^{(0)}$ is open in $\mathcal{F}$ Law12, Lemma $2.31(3)]$, so $\epsilon^{-1}(A)$ is open in $\mathcal{F}$. Since the groupoid $\mathcal{F}$ is topological, $\mathbf{d}: \mathcal{F} \rightarrow$ $\mathcal{F}$ is continuous, so $\mathbf{d}^{-1}\left(\epsilon^{-1}(A)\right)$ is open in $\mathcal{F}$. Also, $\mathcal{F}_{s}$ is open, so

$$
\pi^{-1}\left(\Theta_{0}(s, A)\right)=\mathcal{F}_{s} \cap \mathbf{d}^{-1}\left(\epsilon^{-1}(A)\right)
$$

is open in $\mathcal{F}$ because finite intersections of open sets are open.

We show $\pi: \mathcal{F} \rightarrow \mathcal{G}_{0}$ is open. Fix $s \in S$ and let $T \subseteq_{\text {fin }} s^{\downarrow}$ for an arbitrary basic open set $\mathcal{F}_{s: T}$ in $\mathcal{F}$. Observe

$$
\pi\left(\mathcal{F}_{s: T}\right)=\Theta_{0}\left(s, \epsilon\left(\mathbf{d}\left(\mathcal{F}_{s: T}\right)\right)\right)=\Theta_{0}\left(s, \epsilon\left(\mathcal{F}_{s^{*} s: s^{*} s \mathbf{d}(T)}\right)\right)=\Theta_{0}\left(s, \mathcal{F}_{s^{*} s: s^{*} s \mathbf{d}(T)}\right),
$$

using Lemma 3.2.17.

In the next subsections we consider how $\pi$ restricts to ultrafilters and tight filters. 


\subsubsection{Ultrafilters}

We define groupoid reductions in order to relate $\mathcal{U}$ to a certain subgroupoid of $\mathcal{G}_{0}$ via $\pi$, and we find a nice basis for $\tau_{\text {patch }}$ on $\mathcal{U}$.

Given a groupoid $\mathcal{G}$ and a subset $R$ of $\mathcal{G}^{(0)}$, the reduction of $\mathcal{G}$ by $R$ is

$$
\left.\mathcal{G}\right|_{R}:=\{\gamma \in \mathcal{G}: \mathbf{d}(\gamma), \mathbf{r}(\gamma) \in R\}
$$

with the restricted operations in $\mathcal{G}$, which is a subgroupoid of $\mathcal{G}$ Ren80, Defintion 1.4 , pp. 8]. Recall we identify $\mathcal{G}_{0}^{(0)}$ with $\mathrm{F}(E)$ by $\xi \mapsto[e, \xi]$, where $e \in \xi$. We denote by $\mathcal{G}_{\infty}$ the reduction of $\mathcal{G}_{0}$ by the subset of $\mathcal{G}_{0}^{(0)}$ identifying with $\mathrm{U}(E)$. The groupoid $\mathcal{G}_{\infty}$ is endowed with the subspace topology, having the collection of subsets of the form

$$
\Theta_{\infty}(x, A):=\left\{[x, \xi] \in \mathcal{G}_{\infty}: \xi \in A\right\}
$$

where $x \in S$ and $A \subseteq \mathrm{U}_{x^{*} x}$ is open in $\mathrm{U}(E)$, for a basis. Then, $\mathcal{G}_{\infty}$ is topological.

Corollary 3.4.2. The restriction $\left.\pi\right|_{u}: \mathcal{U} \rightarrow \mathcal{G}_{\infty}$ is a topological isomorphism.

Proof. We show $\pi(\mathcal{U})=\mathcal{G}_{\infty}$. Fix $U \in \mathcal{U}$. Then, $\pi(U)=\left[s, \xi_{U}\right]$, where $s \in U$. Observe $\mathbf{d}(U) \in \mathcal{U}^{(0)}$, so $\xi_{U}=\epsilon(\mathbf{d}(U)) \in \mathbf{U}(E)$ by Proposition 3.3.2. Thus, $\left[s, \xi_{U}\right]$ has source and range identifying with elements in $\mathrm{U}(E)$, so $\pi(U) \in \mathcal{G}_{\infty}$. Now take $[s, \xi] \in \mathcal{G}_{\infty} \subseteq \mathcal{G}_{0}$. As $\pi$ maps $\mathcal{F}$ onto $\mathcal{G}_{0}$, there is $U \in \mathcal{F}$ such that $\pi(U)=[s, \xi]$. Say $t \in U$, so $\pi(U)=\left[t, \xi_{U}\right]=[s, \xi]$. In particular, $\xi=\xi_{U}$, so $\mathrm{U}(E) \ni \xi=\xi_{U}=\epsilon(\mathbf{d}(U))$ and Proposition 3.3 .2 implies $\mathbf{d}(U) \in \mathcal{U}^{(0)}$. Then, $U=U \cdot \mathbf{d}(U) \in \mathcal{U}$ because $\mathcal{U}$ is an ideal in $\mathcal{F}$ (Proposition 3.1.5). Thus, $[s, \xi]=\pi(U) \in \pi(\mathcal{U})$, and so $\pi(\mathcal{U})=\mathcal{G}_{\infty}$

The corollary then follows from $\pi(\mathcal{U})=\mathcal{G}_{\infty}$ since $\pi$ is a topological isomorphism by Theorem 3.4.1.

We use Corollary 3.4 .2 to generalise [LL13, Proposition 5.18], finding a nice basis for $\tau_{\text {patch }}$ on $\mathcal{U}$. For all $x \in S$, let $\mathcal{U}_{x}:=\mathcal{F}_{x} \cap \mathcal{U}$. 
Corollary 3.4.3. The collection $\left(\mathcal{U}_{x}\right)_{x \in S}$ is a basis for $\tau_{\text {patch }}$ on $\mathcal{U}$.

Proof. By Corollary 3.4.2, $\left.\pi\right|_{\mathfrak{u}}$ is a homeomorphism from $\mathcal{U}$ to $\mathcal{G}_{\infty}$, so it suffices to show $\left(\pi\left(\mathcal{U}_{x}\right)\right)_{x \in S}$ is a basis for the topology on $\mathcal{G}_{\infty}$. Observe

$$
\pi\left(\mathcal{U}_{x}\right)=\left\{\left[x, \xi_{U}\right]: U \in \mathcal{U}_{x}\right\}=\left\{[x, \xi]: \xi \in \mathrm{U}_{x^{*} x}\right\}=\Theta_{\infty}\left(x, \mathrm{U}_{x^{*} x}\right),
$$

so $\left(\pi\left(\mathcal{U}_{x}\right)\right)_{x \in S}=\left(\Theta_{\infty}\left(x, \mathrm{U}_{x^{*} x}\right)\right)_{x \in S}$.

We show $\left(\Theta_{\infty}\left(x, \mathrm{U}_{x^{*} x}\right)\right)_{x \in S}$ is a basis for the patch topology on $\mathcal{G}_{\infty}$. Fix $x \in S$ and take any $A \subseteq \mathrm{U}_{x^{*} x}$ so that $A$ is in $\tau_{\text {patch }}$ on $\mathrm{U}(E)$. Then, $\Theta_{\infty}(x, A)$ is an arbitrary basic open subset of $\mathcal{G}_{\infty}$. Recall from Lemma 3.2.6 that $\tau_{\text {patch }}$ coincides with $\tau_{S}$ on $\mathrm{U}(E)$, having $\left(\mathrm{U}_{e}\right)_{e \in E}$ for a basis. Thus, $A=\bigcup_{e \in X} \mathrm{U}_{e}$ for some $X \subseteq E$, so $\Theta_{\infty}(x, A)=\Theta_{\infty}\left(x, \bigcup_{e \in X} \bigcup_{e}\right)$. Let $[x, \xi] \in \Theta_{\infty}\left(x, \bigcup_{e \in X} \bigcup_{e}\right)$. We find $y \in S$ such that

$$
[x, \xi] \in \Theta_{\infty}\left(y, \mathrm{U}_{y^{*} y}\right) \subseteq \Theta_{\infty}\left(x, \bigcup_{e \in X} \mathrm{U}_{e}\right) .
$$

Notice $\xi \in \bigcup_{e \in X} \bigcup_{e}$ means there is $e \in X$ such that $\xi \in \bigcup_{e}$, so

$$
[x, \xi] \in \Theta_{\infty}\left(x, \mathrm{U}_{e}\right) \subseteq \Theta_{\infty}\left(x, \bigcup_{e \in X} \mathrm{U}_{e}\right)
$$

By putting $y:=x e$ and observing that $x^{*} x e \in \xi$ (Lemma 2.1.15), we can show

$$
[x, \xi] \in \Theta_{\infty}\left(y, \mathrm{U}_{y^{*} y}\right) \subseteq \Theta_{\infty}\left(x, \mathrm{U}_{e}\right) \subseteq \Theta_{\infty}\left(x, \bigcup_{e \in X} \mathrm{U}_{e}\right) \subseteq \Theta_{\infty}(x, A)
$$

SO

$$
[x, \xi] \in \Theta_{\infty}\left(y, \mathrm{U}_{y^{*} y}\right) \subseteq \Theta_{\infty}(x, A) .
$$

Therefore, $\left(\pi\left(\mathcal{U}_{x}\right)\right)_{x \in S}=\left(\Theta_{\infty}\left(x, \cup_{x^{*} x}\right)\right)_{x \in S}$ is a basis.

\subsubsection{Exel's tight groupoid}

Define $\mathrm{T}(E)$ to be the closure of $\mathrm{U}(E)$ in $\mathrm{F}(E)$ with respect to $\tau_{\text {patch. }}$. The set $\mathrm{T}(E)$ is that of $\left[\right.$ Law12, Proposition 2.25]. We denote by $\mathcal{G}_{\text {tight }}$ the reduction 
of $\mathcal{G}_{0}$ by the subset of $\mathcal{G}_{0}^{(0)}$ identifying with $\mathrm{T}(E)$. Then, $\mathcal{G}_{\text {tight }}$ is Exel's tight groupoid from Exe08, Theorem 13.3].

Let $\mathcal{T}:=\pi^{-1}\left(\mathcal{G}_{\text {tight }}\right)$. Since $\pi: \mathcal{F} \rightarrow \mathcal{G}_{0}$ is a topological isomorphism and $\mathcal{G}_{\text {tight }}$ is a subgroupoid of $\mathcal{G}_{0}, \mathcal{T}$ is a topological subgroupoid of $\mathcal{F}$, which is topologically isomorphic to Exel's tight groupoid $\mathcal{G}_{\text {tight }}$. By [LL13, Lemma 5.9], $\mathcal{T}$ is the groupoid denoted by $G_{t}(S)$ in [LL13]. Also, $\mathcal{T}$ is the 'reduction' of $\mathcal{L}$ with respect to a certain notion of coverage in [Cas20, Corollary 6.8].

\subsection{Application to self-similar groups}

In the remaining section of the thesis, we use $\pi: \mathcal{F} \rightarrow \mathcal{G}_{0}$ to show how describing filters in inverse semigroups is a matter of describing the filters of idempotents. We apply our results to an inverse semigroup $S_{G, X}$ associated to a given self-similar group $(G, X)$, in which case we have the following specific diagram, where the groupoids $\mathcal{G}_{\text {tight }}, \mathcal{T}, \mathcal{G}_{\infty}$ and $\mathcal{U}$ are all isomorphic.

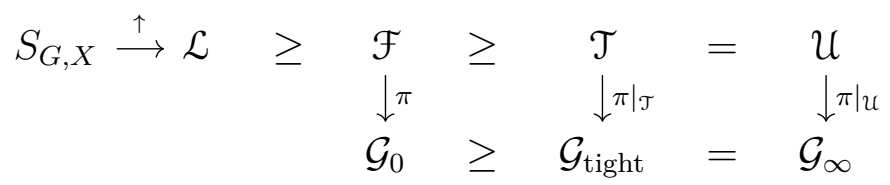

\subsubsection{Filters in terms of filters of idempotents}

In general, describing filters is easier than describing germs. For example, there is no equivalence relation necessary to define filters, unlike germs. However, in practice it can still be difficult to describe the filters in an inverse semigroup because the natural partial order on the inverse semigroup may not be well-behaved. We use $\pi: \mathcal{F} \rightarrow \mathcal{G}_{0}$ to simplify this problem. Recall from Theorem 3.4.1 that the inverse $\pi^{-1}: \mathcal{G}_{0} \rightarrow \mathcal{F}$ of $\pi$ is given by

$$
[s, \xi] \mapsto \pi^{-1}([s, \xi])=(s \xi)^{\uparrow} .
$$

Since $\pi$ is a bijection, the set $\mathcal{F}$ of proper filters in $S$ is given by

$$
\mathcal{F}=\pi^{-1}\left(\mathcal{G}_{0}\right)=\left\{(s \xi)^{\uparrow}: s \in S \text { and } \xi \in \mathrm{F}_{s^{*} s}\right\} .
$$


Equation 3.2 demonstrates how $\pi: \mathcal{F} \rightarrow \mathcal{G}_{0}$ converts the problem of understanding the filters in an inverse semigroup into a problem of understanding the filters of idempotents.

The set $\mathcal{U}$ of ultrafilters in $S$ is given by

$$
\mathcal{U}=\pi^{-1}\left(\mathcal{G}_{\infty}\right)=\left\{(s \xi)^{\uparrow}: s \in S \text { and } \xi \in \mathrm{U}_{s^{*} s}\right\}
$$

using Corollary 3.4 .2 for $\mathcal{U}=\pi^{-1}\left(\mathcal{G}_{\infty}\right)$. Moreover, there is a notion of $E$ being compactable defined in Law10a such that $E$ is compactable if, and only if, $\mathrm{U}(E)=\mathrm{T}(E)$ Law10a, Theorem 2.5]. By definition of groupoid reductions and because $\pi$ is a bijection, we have

$$
\mathcal{U}=\mathcal{T} \Longleftrightarrow \mathrm{U}(E)=\mathrm{T}(E) \Longleftrightarrow \mathcal{G}_{\infty}=\mathcal{G}_{\text {tight }} .
$$

Therefore, when $E$ is compactable, we have

$$
\mathcal{T}=\mathcal{U}=\pi^{-1}\left(\mathcal{G}_{\infty}\right)=\left\{(s \xi)^{\uparrow}: s \in S \text { and } \xi \in \mathrm{U}_{s^{*} s}\right\}
$$

\subsubsection{Filters associated to self-similar groups}

For the remainder of the thesis, we utilise the results of the previous section based on the topological isomorphism $\pi: \mathcal{F} \rightarrow \mathcal{G}_{0}$ in order to describe the filters in inverse semigroups associated to self-similar groups. Recall from the introduction that studying self-similar groups is interesting in part because of the class of $\mathrm{C}^{*}$-algebras generated by self-similar groups.

Recall that an inverse semigroup is a group if, and only if, there is exactly one idempotent, in which case the idempotent is called the identity and denoted by $e$ Law98, Proposition 1.4.4, pp. 19]. We refer the reader to [DF04] for the following rudiments of group theory. Let $Y$ be a set. We denote the set of bijections of $Y$ by $S(Y)$. The set $S(Y)$ under function composition forms a group. An action of a group $G$ on the set $Y$ is a homomorphism $\phi: G \rightarrow S(Y)$. Given $(g, y) \in G \times Y$, we write $g \cdot y:=\phi(g)(y)$. If $\phi$ is injective, then we say $\phi$ is faithful. 
Let $X$ be a finite set. We write $X^{n}$ for the set of words in $X$ of length $n$ (that is, $n$-tuples denoted by concatenation), with $X^{0}=\{\varnothing\}$, and $X^{*}:=$ $\bigcup_{n \geq 0} X^{n}$. We denote by $X^{\omega}$ the set of infinite words in $X$. For all $\alpha, \beta \in X^{*}$, we define $\alpha \preceq \beta$ if there is $\epsilon \in X^{*}$ such that $\alpha \epsilon=\beta$. The relation $\preceq$ is a partial order on $X^{*}$. A faithful group action of $G$ on $X^{*}$ is self-similar if, for all $g \in G$ and all $x \in X$, there are $h \in G$ and $y \in X$ such that

$$
g \cdot(x \alpha)=y(h \cdot \alpha)
$$

for all $\alpha \in X^{*}$ Nek05, Definition 1.5.1, pp. 10]. As in [Lac+14, §2], we assume $g \cdot \varnothing=\varnothing$, for all $g \in G$. We say such a pair $(G, X)$ is a self-similar group. The following results can be found in Nek05, Lac+14].

Lemma 3.5.1. Let $(G, X)$ be a self-similar group. There is a map from $G \times X$ to $G$ denoted by

$$
\left.(g, x) \mapsto g\right|_{x}
$$

such that $g \cdot x \in X$ and $g \cdot(x \alpha)=(g \cdot x)\left(\left.g\right|_{x} \cdot \alpha\right)$, for all $\alpha \in X^{*}$.

Proof. Fix $(g, x) \in G \times X$. There exists $(h, y) \in G \times X$ such that $g \cdot(x \alpha)=$ $y(h \cdot \alpha)$, for all $\alpha \in X^{*}$. In order to define $\left.g\right|_{x}$ we show $(h, y)$ is unique. Suppose also that $(i, z) \in G \times X$ satisfies $g \cdot(x \alpha)=z i \cdot \alpha$, for all $\alpha \in X^{*}$. Thus, $y h \cdot \alpha=z i \cdot \alpha$, for all $\alpha \in X^{*}$. Since $y, z \in X^{1}$, we have both $y=z$ and $h \cdot \alpha=i \cdot \alpha$, for all $\alpha \in X^{*}$. Hence, $\phi(h)=\phi(i)$, and the action is faithful by definition, so $h=i$. Therefore, $(h, y)=(i, z)$. We denote $h$ by $\left.g\right|_{x}$, and we can see $y=g \cdot x$ by setting $w:=\varnothing$ because $h \cdot \varnothing=\varnothing$.

Due to Lemma 3.5.1, we can redefine self-similar groups as follows. A faithful group action of $G$ on $X^{*}$ is self-similar if there is a map $\left.(g, x) \mapsto g\right|_{x}$ from $G \times X$ to $G$ such that, for every $(g, x) \in G \times X$,

$$
g \cdot(x \alpha)=(g \cdot x)\left(\left.g\right|_{x} \cdot \alpha\right)
$$

for all $\alpha \in X^{*}$. We extend $\left.(g, x) \mapsto g\right|_{x}$ from $G \times X$ to $G$ in $(G, X)$ to a map from $G \times X^{*}$ as follows. Define $\left.g\right|_{\varnothing}:=g$. Given $w_{1} w_{2} \ldots w_{n} \in X^{*}$ for some 
$n \in \mathbb{N}$, where each $w_{i}$ is in $X$, define

$$
\left.g\right|_{w_{1} w_{2} \ldots w_{n}}:=\left.\left.\left.g\right|_{w_{1}}\right|_{w_{2}} \cdots\right|_{w_{n}} .
$$

The map $\left.(g, \alpha) \mapsto g\right|_{\alpha}$ from $G \times X^{*}$ to $G$ is called the restriction.

We collate further properties of self-similar groups as in Nek05, Lac+14.

Lemma 3.5.2. Let $(G, X)$ be a self-similar group. Then, the following hold.

(1) For all $g \in G$, for all $\alpha \in X^{*}, g \cdot(\alpha \beta)=(g \cdot \alpha)\left(\left.g\right|_{\alpha} \cdot \beta\right)$, for all $\beta \in X^{*}$.

(2) For all $g \in G, g \cdot \varnothing=\varnothing$ and $\left.g\right|_{\varnothing}=g$.

(3) For all $\alpha \in X^{*}, e \cdot \alpha=\alpha$ and $\left.e\right|_{\alpha}=e$.

(4) For all $g \in G$ and for all $\alpha, \beta \in X^{*},\left.g\right|_{\alpha \beta}=\left.\left(\left.g\right|_{\alpha}\right)\right|_{\beta}$.

(5) For all $g, h \in G$ and for all $\alpha \in X^{*},\left.(g h)\right|_{\alpha}=\left.\left.g\right|_{h \cdot \alpha} h\right|_{\alpha}$.

(6) For all $g \in G$ and for all $\alpha \in X^{*},\left(\left.g\right|_{\alpha}\right)^{-1}=\left.\left(g^{-1}\right)\right|_{g \cdot \alpha}$.

(7) For all $g \in G$ and for all $\alpha \in X^{*},|\alpha|=|g \cdot \alpha|$.

Proof. (1) This can be shown to hold by induction on the length of the variable $\alpha$.

(2) Recall $g \cdot \varnothing=\varnothing$ holds by definition of a self-similar group. Then, (1) implies,

$$
g \cdot \beta=g \cdot(\varnothing \beta)=(g \cdot \varnothing)\left(\left.g\right|_{\varnothing} \cdot \beta\right)=\left.g\right|_{\varnothing} \cdot \beta,
$$

for all $\beta \in X^{*}$. As the group action is faithful, it follows that $g=\left.g\right|_{\varnothing}$.

(3) The groupoid identity $e$ in $G$ gives the identity bijection of $X^{*}$ because the group action is a homomorphism. Hence, $e \cdot \alpha=\alpha$. Using the above and by (1), we have

$$
\alpha(e \cdot \beta)=\alpha \beta=e \cdot(\alpha \beta)=(e \cdot \alpha)\left(\left.e\right|_{\alpha} \cdot \beta\right)=\alpha\left(\left.e\right|_{\alpha} \cdot \beta\right),
$$

so $e \cdot \beta=\left.e\right|_{\alpha} \cdot \beta$, for all $\beta \in X^{*}$. Since the action is faithful, it follows that $\left.e\right|_{\alpha}=e$. 
(4) This is immediate from our definition of the restriction.

(5) Observe that, for all $\beta \in X^{*}$,

$$
\begin{aligned}
((g h) \cdot \alpha)\left(\left.(g h)\right|_{\alpha} \cdot \beta\right) & =(g h) \cdot(\alpha \beta) \\
& =g \cdot(h \cdot(\alpha \beta)) \\
& =g \cdot\left((h \cdot \alpha)\left(\left.h\right|_{\alpha} \cdot \beta\right)\right) \\
& =(g \cdot(h \cdot \alpha))\left(\left.g\right|_{h \cdot \alpha} \cdot\left(\left.h\right|_{\alpha} \cdot \beta\right)\right) \\
& =((g h) \cdot \alpha)\left(\left(\left.\left.g\right|_{h \cdot \alpha} h\right|_{\alpha}\right) \cdot \beta\right) .
\end{aligned}
$$

Thus,

$$
\left.(g h)\right|_{\alpha} \cdot \beta=\left(\left.\left.g\right|_{h \cdot \alpha} h\right|_{\alpha}\right) \cdot \beta,
$$

for all $\beta \in X^{*}$. Because the action of $G$ on $X^{*}$ is faithful, it follows that $\left.(g h)\right|_{\alpha}=\left.\left.g\right|_{h \cdot \alpha} h\right|_{\alpha}$.

(6) Take any $\beta \in X^{*}$. Observe

$$
\left.\left(g^{-1}\right)\right|_{g \cdot \alpha} \cdot \beta=\left(\left.\left(g^{-1}\right)\right|_{g \cdot \alpha} e\right) \cdot \beta=\left(\left.\left(g^{-1}\right)\right|_{g \cdot \alpha}\left(\left.g\right|_{\alpha}\left(\left.g\right|_{\alpha}\right)^{-1}\right)\right) \cdot \beta
$$

and $\left.\left.g^{-1}\right|_{g \cdot \alpha} g\right|_{\alpha}=\left.\left(g^{-1} g\right)\right|_{\alpha}=\left.e\right|_{\alpha}=e$ by (5) and (3). So

$$
\left(\left.\left.\left.g^{-1}\right|_{g \cdot \alpha} g\right|_{\alpha} g\right|_{\alpha} ^{-1}\right) \cdot \beta=\left(\left.e g\right|_{\alpha} ^{-1}\right) \cdot \beta=\left.g\right|_{\alpha} ^{-1} \cdot \beta .
$$

Therefore, $\left.\left(g^{-1}\right)\right|_{g \cdot \alpha} \cdot \beta=\left(\left.g\right|_{\alpha}\right)^{-1} \cdot \beta$, for all $\beta \in X^{*}$. Because the group action is faithful, we have $\left.\left(g^{-1}\right)\right|_{g \cdot \alpha}=\left(\left.g\right|_{\alpha}\right)^{-1}$.

(7) Recall from Lemma 3.5.1 that $g \cdot x \in X$, which implies $|g \cdot x|=|x|$, for all $g \in G$ and for all $x \in X$. It follows from (1) and a proof by induction on the size of $\alpha$ that $|g \cdot \alpha|=|\alpha|$, for all $g \in G$ and $\alpha \in X^{*}$.

Given a self-similar group, we construct an inverse semigroup as per EP17, 4.1. Definition]. Let $(G, X)$ be a self-similar group. We will define the set and binary operation that will be the inverse semigroup $S_{G, X}$ associated to $(G, X)$. Then, we will show $S_{G, X}$ is a regular semigroup whose idempotents commute and conclude from Theorem 2.1.7 that $S_{G, X}$ is an inverse semigroup. 
Define

$$
S_{G, X}:=\left(X^{*} \times G \times X^{*}\right) \cup\{0\} .
$$

The elements of $X^{*} \times G \times X^{*}$ are nonzero. For all nonzero $(\alpha, g, \beta),(\gamma, h, \delta) \in$ $S_{G, X}$, we define

$$
(\alpha, g, \beta)(\gamma, h, \delta):= \begin{cases}\left(\alpha g \cdot \epsilon,\left.g\right|_{\epsilon} h, \delta\right) & \text { if } \exists \epsilon \in X^{*}(\beta \epsilon=\gamma), \\ \left(\alpha, g\left(\left.\left(h^{-1}\right)\right|_{\epsilon}\right)^{-1}, \delta\left(h^{-1} \cdot \epsilon\right)\right) & \text { if } \exists \epsilon \in X^{*}(\beta=\gamma \epsilon), \\ 0 & \text { otherwise, }\end{cases}
$$

as per [EP17, Definition 4.1]. Consider the map $S_{G, X} \times S_{G, X} \rightarrow S_{G, X}$ defined by

$$
(s, t) \mapsto s \circ t:= \begin{cases}s t & \text { if } s \text { and } t \text { are nonzero, } \\ 0 & \text { otherwise. }\end{cases}
$$

For brevity we will write st instead of $s \circ t$.

Lemma 3.5.3. The set $S_{G, X}$ with $(s, t) \mapsto$ st is a semigroup.

Proof. We show $s(t u)=(s t) u$, for all $s, t, u \in S_{G, X}$. If any of $s, t$ or $u$ are 0 , then $s(t u)=0=(s t) u$. Otherwise, $s, t, u \in X^{*} \times G \times X^{*}$, so we write $s=(\alpha, g, \beta), t=(\gamma, h, \delta)$ and $u=(\epsilon, i, \kappa)$. If neither $\beta \preceq \gamma$ nor $\gamma \preceq \beta$, then $(s t) u=0 u=0$, so we want $s(t u)=0$. If $t u=0$, then $s(t u)=s 0=0$, so we are done. If $t u$ is nonzero, the first component of $t u$ is either $\gamma$ or $\gamma \lambda$ for some $\lambda \in X^{*}$. When the first component of $t u$ is $\gamma$, the fact that neither $\beta \preceq \gamma$ nor $\gamma \preceq \beta$ means $s(t u)=0$, as required; when the first component of $t u$ is $\gamma \lambda$, each of $\beta \preceq \gamma \lambda$ and $\gamma \lambda \preceq \beta$ would imply either $\beta \preceq \gamma$ or $\gamma \preceq \beta$. That is, 'neither $\beta \preceq \gamma$ nor $\gamma \preceq \beta$ ' would not hold, which would be a contradiction. Hence, neither $\beta \preceq \gamma \lambda$ nor $\gamma \lambda \preceq \beta$, and so $s(t u)=0$, as wanted. Therefore, 'neither $\beta \preceq \gamma$ nor $\gamma \preceq \beta$ ' implies $s(t u)=0=(s t) u$. Similarly, 'neither $\delta \preceq \epsilon$ nor $\epsilon \preceq \delta$ ' implies $s(t u)=0=(s t) u$.

Having traversed the 'trivial cases', what remains is the situation where both either $\beta \preceq \gamma$ or $\gamma \preceq \beta$ and either $\delta \preceq \epsilon$ or $\epsilon \preceq \delta$. That is, one of the following cases holds: 
(1) $\beta \preceq \gamma$ and $\delta \preceq \epsilon$,

(2) $\beta \preceq \gamma$ and $\epsilon \preceq \delta$,

(3) $\gamma \preceq \beta$ and $\delta \preceq \epsilon$, or

(4) $\gamma \preceq \beta$ and $\epsilon \preceq \delta$.

Suppose (1) holds. Let $\lambda, \mu \in X^{*}$ satisfy $\beta \lambda=\gamma$ and $\delta \mu=\epsilon$. Using $\lambda$ and $\mu$, we can compute

$$
\begin{aligned}
& (s t) u=((\alpha, g, \beta)(\gamma, h, \delta))(\epsilon, i, \kappa)=\left(\alpha(g \cdot \lambda)\left(\left(\left.g\right|_{\lambda} h\right) \cdot \mu\right),\left.\left(\left.g\right|_{\lambda} h\right)\right|_{\mu} i, \kappa\right), \text { and } \\
& s(t u)=(\alpha, g, \beta)((\gamma, h, \delta)(\epsilon, i, \kappa))=\left(\alpha(g \cdot(\lambda(h \cdot \mu))),\left.\left.g\right|_{\lambda(h \cdot \mu)} h\right|_{\mu} i, \kappa\right) .
\end{aligned}
$$

To complete the proof, it suffices to show $(g \cdot \lambda)\left(\left.g\right|_{\lambda} h \cdot \mu\right)=g \cdot(\lambda(h \cdot \mu))$ and $\left.\left(\left.g\right|_{\lambda} h\right)\right|_{\mu}=\left.\left.g\right|_{\lambda(h \cdot \mu)} h\right|_{\mu}$. Observe

$$
g \cdot(\lambda(h \cdot \mu))=(g \cdot \lambda)\left(\left.g\right|_{\lambda} \cdot(h \cdot \mu)\right)=(g \cdot \lambda)\left(\left(\left.g\right|_{\lambda} h\right) \cdot \mu\right),
$$

using Lemma 3.5.2(1) for the first equality. Also,

$$
\left.\left(\left.g\right|_{\lambda} h\right)\right|_{\mu}=\left.\left.\left.g\right|_{\lambda}\right|_{h \cdot \mu} h\right|_{\mu}=\left.\left.g\right|_{\lambda(h \cdot \mu)} h\right|_{\mu},
$$

using Lemma 3.5.2(5) and 3.5.2(4), respectively.

The other cases are similar.

Lemma 3.5.4. The semigroup $S_{G, X}$ is regular.

Proof. Fix $(\alpha, g, \beta) \in S_{G, X}$. Using various properties from Lemma 3.5.2, we have

$$
\begin{aligned}
(\alpha, g, \beta)\left(\beta, g^{-1}, \alpha\right)(\alpha, g, \beta) & =\left(\alpha(g \cdot \varnothing),\left.g\right|_{\varnothing} g^{-1}, \alpha\right)(\alpha, g, \beta) \\
& =\left(\alpha \varnothing, g g^{-1}, \alpha\right)(\alpha, g, \beta) \\
& =(\alpha, e, \alpha)(\alpha, g, \beta) \\
& =\left(\alpha(e \cdot \varnothing),\left.e\right|_{\varnothing} g, \beta\right) \\
& =(\alpha \varnothing, e g, \beta) \\
& =(\alpha, g, \beta),
\end{aligned}
$$


So

$$
(\alpha, g, \beta)\left(\beta, g^{-1}, \alpha\right)(\alpha, g, \beta)=(\alpha, g, \beta)
$$

Similarly, we can show

$$
\left(\beta, g^{-1}, \alpha\right)(\alpha, g, \beta)\left(\beta, g^{-1}, \alpha\right)=\left(\beta, g^{-1}, \alpha\right)
$$

Therefore, $\left(\beta, g^{-1}, \alpha\right)$ is an inverse of $(\alpha, g, \beta)$. Moreover, 0 has itself for an inverse. Thus, every element in $S_{G, X}$ has an inverse.

For all $\alpha \in X^{*}$, define $f_{\alpha}:=(\alpha, e, \alpha)$.

Lemma 3.5.5 (Equation (4.4) of [EP17]). The set $E\left(S_{G, X}\right)$ of idempotents in $S_{G, X}$ is

$$
E\left(S_{G, X}\right)=\left\{f_{\alpha}: \alpha \in X^{*}\right\} \cup\{0\}
$$

Proof. Properties of self-similar groups from Lemma 3.5.2 make it routine to show $E\left(S_{G, X}\right) \supseteq\left\{f_{\alpha}: \alpha \in X^{*}\right\} \cup\{0\}$.

Fix $e \in E\left(S_{G, X}\right)$. If $e=0$, then we are done. Otherwise, write $e=$ $(\alpha, g, \beta)$. Then, $(\alpha, g, \beta)(\alpha, g, \beta)=(\alpha, g, \beta) \neq 0$ implies either $\beta \preceq \alpha$ or $\alpha \preceq \beta$. Suppose $\beta \preceq \alpha$ and find $\gamma \in X^{*}$ so that $\beta \gamma=\alpha$. Thus,

$$
(\alpha, g, \beta)=(\alpha, g, \beta)(\alpha, g, \beta)=\left(\alpha(g \cdot \gamma),\left.g\right|_{\gamma} g, \beta\right)
$$

It follows that $g \cdot \gamma=\varnothing$, so

$$
\gamma=e \cdot \gamma=\left(g^{-1} g\right) \cdot \gamma=g^{-1} \cdot(g \cdot \gamma)=g^{-1} \cdot \varnothing=\varnothing
$$

Hence, $\alpha=\beta$. Moreover, $\left.g\right|_{\gamma}=\left.g\right|_{\varnothing}=g$, so $g=\left.g\right|_{\gamma} g=g g$ implies $g=e$. Therefore, $(\alpha, g, \beta)=f_{\alpha}$. A similar argument applies in case $\alpha \preceq \beta$, so we have $E\left(S_{G, X}\right) \subseteq\left\{f_{\alpha}: \alpha \in X^{*}\right\} \cup\{0\}$.

Lemma 3.5.6 (Proposition 4.6 of EP17]). For all $\alpha, \beta \in X^{*}$,

$$
\alpha \preceq \beta \Longleftrightarrow f_{\alpha} f_{\beta}=f_{\beta} f_{\alpha}=f_{\beta}
$$


Proof. Suppose $\alpha \preceq \beta$. Find $\gamma \in X^{*}$ so that $\alpha \gamma=\beta$. Compute

$$
f_{\alpha} f_{\beta}=(\alpha, e, \alpha)(\beta, e, \beta)=\left(\alpha(e \cdot \gamma),\left.e\right|_{\gamma} e, \beta\right)=(\alpha \gamma, e e, \beta)=(\beta, e, \beta)=f_{\beta}
$$

A similar computation shows $f_{\beta} f_{\alpha}=f_{\beta}$. Therefore, $f_{\alpha} f_{\beta}=f_{\beta} f_{\alpha}=f_{\beta}$.

Suppose $f_{\alpha} f_{\beta}=f_{\beta} f_{\alpha}=f_{\beta}$. In particular, $f_{\beta} f_{\alpha}=f_{\beta}$, so $f_{\beta} f_{\alpha}$ is nonzero. Then, either $\alpha \preceq \beta$ or $\beta \preceq \alpha$. If the former holds, then we are done. Otherwise $\beta \preceq \alpha$. Find $\gamma \in X^{*}$ so that $\beta \gamma=\alpha$. Then, $f_{\beta} f_{\alpha}=f_{\beta}$ implies

$$
f_{\beta}=f_{\beta} f_{\alpha}=(\beta, e, \beta)(\alpha, e, \alpha)=\left(\beta(e \cdot \gamma),\left.e\right|_{\gamma} e, \alpha\right)=f_{\alpha},
$$

so $\alpha=\beta$. In particular, $\alpha \preceq \beta$ because $\preceq$ is a partial order.

Proposition 3.5.7 (Proposition 4.3 of [EP17]). The semigroup $S_{G, X}$ is an inverse semigroup with zero.

Proof. It is a consequence of Lemmas 3.5.5 and 3.5.6 that idempotents commute. Moreover, Lemma 3.5 .4 tells us that $S_{G, X}$ is a regular semigroup. Thus, $S_{G, X}$ is an inverse semigroup with zero by the characterisation of inverse semigroups given in Theorem 2.1.7.

Recall filters in $S_{G, X}$ are nonempty down-directed up-sets with respect to the natural partial order. Describing the natural partial order on $S_{G, X}$ is messy, so filters in $S_{G, X}$ are hard to handle. We restrict our attention to $E\left(S_{G, X}\right)$. Notice that Lemma 3.5.6 implies

$$
\alpha \preceq \beta \Longleftrightarrow f_{\beta} \leq f_{\alpha}
$$

for all $\alpha, \beta \in X^{*}$ EP16, Equation (4.7)]. That is, unlike $S_{G, X}$, describing the natural partial order on $E\left(S_{G, X}\right)$ is clean, so filters in $E\left(S_{G, X}\right)$ are easy to handle.

Lemma 3.5.8 (§8 of [EP17]). The sets $\mathrm{U}\left(E\left(S_{G, X}\right)\right)$ and $\mathrm{T}\left(E\left(S_{G, X}\right)\right)$ satisfy

$$
\mathrm{T}\left(E\left(S_{G, X}\right)\right)=\mathrm{U}\left(E\left(S_{G, X}\right)\right)=\left\{\left(f_{\left.\xi\right|_{n}}\right)_{n \in \mathbb{N}}: \xi \in X^{\omega}\right\}
$$


Proof. The equation $\mathrm{T}\left(E\left(S_{G, X}\right)\right)=\mathrm{U}\left(E\left(S_{G, X}\right)\right)$ is given in [EP17, pp. 1074].

We show $\mathrm{U}\left(E\left(S_{G, X}\right)\right)=\left\{\left(f_{\left.\xi\right|_{n}}\right)_{n \in \mathbb{N}}: \xi \in X^{\omega}\right\}$. Fix $\xi \in \mathrm{F}\left(E\left(S_{G, X}\right)\right)$. Recall from Section 2.2 .2 that a chain is a totally ordered subset of a partially ordered set. Using the down-directedness of $\xi$, we can show $\xi$ is a chain. Then, since $\xi$ is an up-set, either $\xi=\left(f_{\left.\alpha\right|_{n}}\right)_{n \leq|\alpha|}$ for some $\alpha \in X^{*}$ or $\xi=$ $\left(f_{\left.\eta\right|_{n}}\right)_{n \in \mathbb{N}}$ for some $\eta \in X^{\omega}$. Conversely, subsets of $E\left(S_{G, X}\right)$ of the former or latter form are indeed in $\mathrm{F}\left(E\left(S_{G, X}\right)\right)$. Therefore,

$$
\mathrm{F}\left(E\left(S_{G, X}\right)\right)=\left\{\left(f_{\left.\alpha\right|_{n}}\right)_{n \leq|\alpha|}: \alpha \in X^{*}\right\} \cup\left\{\left(f_{\left.\eta\right|_{n}}\right)_{n \in \mathbb{N}}: \eta \in X^{\omega}\right\} .
$$

It is then routine to show

$$
\mathrm{U}\left(E\left(S_{G, X}\right)\right)=\left\{\left(f_{\left.\eta\right|_{n}}\right)_{n \in \mathbb{N}}: \eta \in X^{\omega}\right\}
$$

completing the proof.

We conclude by contributing to the study of self-similar groups a concrete description of the tight filters and ultrafilters in the inverse semigroup of a given self-similar group, which complements analogous efforts to describe $\mathcal{G}_{\text {tight }}$ concretely using the germ approach in EP17.

Given $(\alpha, \xi) \in X^{*} \times X^{\omega}$, we write $\alpha \prec \xi$ if there is $n \in \mathbb{N}$ so that $\alpha=\left.\xi\right|_{n}$.

Theorem 3.5.9. The sets $\mathfrak{U}\left(S_{G, X}\right)$ and $\mathcal{T}\left(S_{G, X}\right)$ satisfy

$\mathcal{T}\left(S_{G, X}\right)=\mathcal{U}\left(S_{G, X}\right)=\left\{\left((\alpha, g, \beta)\left(f_{\left.\xi\right|_{n}}\right)_{n \in \mathbb{N}}\right)^{\uparrow}:(\alpha, g, \beta) \in S_{G, X}, \beta \prec \xi \in X^{\omega}\right\}$.

Proof. Since $\mathrm{T}\left(E\left(S_{G, X}\right)\right)=\mathrm{U}\left(E\left(S_{G, X}\right)\right)$ by Lemma 3.5.8, Equations 3.3. and 3.4 imply

$$
\mathcal{T}\left(S_{G, X}\right)=\mathcal{U}\left(S_{G, X}\right)=\left\{((\alpha, g, \beta) \eta)^{\uparrow}:(\alpha, g, \beta) \in S_{G, X} \text { and } \eta \in \mathrm{U}_{f_{\beta}}\right\} .
$$

Fix $(\alpha, g, \beta) \in S_{G, X}$. It remains to show $\eta \in \mathrm{U}_{f_{\beta}}$ if, and only if, $\eta=\left(f_{\left.\xi\right|_{n}}\right)_{n \in \mathbb{N}}$, where $\beta \prec \xi \in X^{\omega}$. Suppose $\eta \in \mathrm{U}_{f_{\beta}}$. Lemma 3.5 .8 gives us $\xi \in X^{\omega}$ such that $\eta=\left(f_{\left.\xi\right|_{n}}\right)_{n \in \mathbb{N}}$. Then, $f_{\beta} \in\left(f_{\left.\xi\right|_{n}}\right)_{n \in \mathbb{N}}$, so there is $n \in \mathbb{N}$ such that $f_{\beta}=f_{\left.\xi\right|_{n}}$, in which case $\beta=\left.\xi\right|_{n} \prec \xi$. Now suppose $\eta=\left(f_{\left.\xi\right|_{n}}\right)_{n \in \mathbb{N}}$ for some $\xi \in X^{\omega}$ with $\beta \prec \xi$. Lemma 3.5 .8 implies $\eta$ is in $\mathrm{U}\left(E\left(S_{G, X}\right)\right)$, and $\beta \prec \xi$ means $\beta=\left.\xi\right|_{n}$ for some $k \in \mathbb{N}$. Then, $f_{\beta}=f_{\left.\xi\right|_{k}} \in\left(f_{\left.\xi\right|_{n}}\right)_{n \in \mathbb{N}}=\eta$. Therefore, $\eta \in \mathrm{U}_{f_{\beta}}$. 


\section{Bibliography}

[Arm+20] Armstrong, B., Clark, L. O., an Huef, A., Jones, M., and Lin, Y.-F. Filtering germs: groupoids associated to inverse semigroups. 2020. arXiv: 2010.16113 [math.RA].

[Bic19] Bice, T. An algebraic approach to the Weyl groupoid. 2019. arXiv: 1911.08812 [math.0A]

[BS19] Bice, T. and Starling, C. General non-commutative locally compact locally Hausdorff Stone duality. In: Advances in Mathematics 341 (2019), pp. 40 -91. DOI: https://doi.org/10.1016 /j.aim.2018.10.031.

[Bra27] Brandt, H. Über eine Verallgemeinerung des Gruppenbegriffes. In: Mathematische Annalen 96.1 (1927), pp. 360-366. DOI: 10.1 007/BF01209171

[Cas20] de Castro, G. G. Coverages on Inverse Semigroups. 2020. arXiv: 2005.08714

[DF04] Dummit, D. S. and Foote, R. M. Abstract Algebra. Third. John Wiley and Sons, Inc., 2004. ISBN: 0-471-43334-9.

[Ehr57] Ehresmann, C. Gattungen von lokalen Strukturen. In: Jahresbericht Deutsch. Math.-Verein. 60.Abt. 1 (1957), pp. 49-77.

[Ehr60] Ehresmann, C. Catégories inductives et pseudo-groupes. In: Ann. Inst. Fourier (Grenoble) 10 (1960), pp. 307-332. 
[Exe09] Exel, R. Tight representations of semilattices and inverse semigroups. In: Semigroup Forum 79.1 (2009), pp. 159-182. DOI: 10 $.1007 / \mathrm{s} 00233-009-9165-\mathrm{x}$.

[Exe08] Exel, R. Inverse semigroups and combinatorial $C^{*}$-algebras. In: Bull. Braz. Math. Soc. (N.S.) 39.2 (2008), pp. 191-313. DOI: $10.1007 / \mathrm{s} 00574-008-0080-7$.

[EP16] Exel, R. and Pardo, E. The tight groupoid of an inverse semigroup. In: Semigroup Forum 92.1 (2016), pp. 274-303. DOI: 10 $.1007 / \mathrm{s} 00233-015-9758-5$.

[EP17] Exel, R. and Pardo, E. Self-similar graphs, a unified treatment of Katsura and Nekrashevych $\mathrm{C}^{*}$-algebras. In: Advances in Mathematics 306 (2017), pp. 1046 -1129. DOI: 10.1016/j .aim. 2016 .10 .030 .

[Hah78] Hahn, P. Haar Measure for Measure Groupoids. In: Transactions of the American Mathematical Society 242 (1978), pp. 1-33.

[Hal60] Halmos, P. R. Naive Set Theory. D. van Nostrand Company, Inc., 1960.

[How76] Howie, J. M. An Introduction to Semigroup Theory. Academic Press Inc. (London) Ltd., 1976. ISBN: 75-46333.

[Lac+14] Laca, M., Raeburn, I., Ramagge, J., and Whittaker, M. F. Equilibrium states on the Cuntz-Pimsner algebras of self-similar actions. In: J. Funct. Anal. 266.11 (2014), pp. 6619-6661. DOI: 10 $.1016 / \mathrm{j} \cdot \mathrm{jfa} .2014 .03 .003$.

[Law98] Lawson, M. V. Inverse Semigroups, the Theory of Partial Symmetries. World Scientific Pub Co Inc, 1998. ISBN: 9810233167.

[Law10a] Lawson, M. V. Compactable semilattices. In: Semigroup Forum 81.1 (2010), pp. 187-199. DOI: 10.1007/s00233-010-9245-y. 
[Law93] Lawson, M. V. Coverings and embeddings of inverse semigroups. In: Proc. Edinburgh Math. Soc. 36.3 (1993), pp. 399-419. DOI: 10.1017/S0013091500018502.

[Law10b] Lawson, M. V. A noncommutative generalization of the Stone duality. In: J. Australian Math. Soc. 88.3 (2010). DOI: 10.1017 /S1446788710000145.

[Law12] Lawson, M. V. Non-commutative Stone duality: inverse semigroups, topological groupoids and $\mathrm{C}^{*}$-algebras. In: Int. J. Algebra and Computation 22.6 (2012). DOI: 10.1142/S02181967125 00580 .

[LL13] Lawson, M. V. and Lenz, D. H. Pseudogroups and their étale groupoids. In: Advances in Mathematics 244 (2013), pp. 117 170. DOI: $10.1016 /$ j.aim.2013.04.022

[LMS13] Lawson, M. V., Margolis, S. W., and Steinberg, B. The étale groupoid of an inverse semigroup as a groupoid of filters. In: $J$. Australian Math. Soc. 94.2 (2013), pp. 234-256. DOI: 10.1017 /S144678871200050X.

[Len08] Lenz, D. H. On an order-based construction of a topological groupoid from an inverse semigroup. In: Proc. Edinburgh Math. Soc. 51.2 (2008), pp. 387-406. DOI: 10.1017/S00130915060000 83.

[Mun00] Munkres, J. R. Topology. 2nd ed. Prentice Hall, Inc., 2000. ISBN: 0-13-181629-2.

[Nek05] Nekrashevych, V. Self-Similar Groups. Mathematical surveys and monographs. American Mathematical Society, 2005. ISBN: 9780-8218-3831-0.

[Pat99] Paterson, A. Groupoids, Inverse Semigroups, and their Operator Algebras. Vol. 170. Progress in Mathematics. Birkhäuser Basel, 
1999. ISBN: 978-1-4612-7276-2. DOI: 10.1007/978-1-4612-177 4-9.

[Pet84] Petrich, M. Inverse semigroups. Pure and Applied Mathematics (New York). John Wiley \& Sons, Inc., New York, 1984. ISBN: 0-471-87545-7.

[Pre54a] Preston, G. B. Inverse Semi-Groups. In: Journal of the London Mathematical Society s1-29.4 (1954), pp. 396-403. DOI: 10.1 112 / jlms / s1-29.4.396. eprint: https : / / londmathsoc. onlinelibrary.wiley.com/doi/pdf/10.1112/jlms/s1-29 .4 .396 .

[Pre54b] Preston, G. B. Inverse Semi-Groups with Minimal Right Ideals. In: Journal of the London Mathematical Society s1-29.4 (1954), pp. 404-411. DOI: $10.1112 / \mathrm{jlms} / \mathrm{s} 1-29.4 .404$. eprint: https : //londmathsoc.onlinelibrary.wiley.com/doi/pdf/10.1112 $/ j \operatorname{lms} / \mathrm{s} 1-29.4 .404$.

[Pre54c] Preston, G. B. Representations of Inverse Semi-Groups. In: Journal of the London Mathematical Society s1-29.4 (1954), pp. 411419. DOI: $10.1112 / \mathrm{jlms} / \mathrm{s} 1-29.4 .411$. eprint: https : // londmathsoc . onlinelibrary . wiley . com/doi/pdf /10.1112 /jlms/s1-29.4.411.

[Ren80] Renault, J. A groupoid approach to $\mathrm{C}^{*}$-algebras. Lecture notes in mathematics. Springer, 1980. ISBN: 978-3-540-09977-2. DOI: 10.1007/BFb0091072.

[Res10] Resende, P. Lectures on etale groupoids, inverse semigroups and quantales. 2010.

[Sie34] Sierpiński, W. F. Introduction to general topology. The University of Toronto Press, 1934.

[Sim18] Sims, A. Hausdorff étale groupoids and their $\mathrm{C}^{*}$-algebras. 2018. arXiv: 1710.10897 [math.0A]. 
[UT18] Ultrafilter Theorem. 2018 (accessed 5 November 2020). URL: https://ncatlab.org/nlab/show/ultrafilter+theorem.

[Vag52] Vagner, V. V. Generalized groups. In: Doklady Akad. Nauk SSSR (N.S.) 84 (1952), pp. 1119-1122.

[Vag53] Vagner, V. V. The theory of generalized heaps and generalized groups. In: Mat. Sbornik N.S. 32(74) (1953), pp. 545-632. 